\title{
Differentiability with respect to initial functions of solutions to nonlinear hyperbolic functional differential systems
}

\author{
Zdzislaw Kamont
}

Received: 1 March 2012 / Accepted: 7 November 2013 / Published online: 18 December 2013

(C) The Author(s) 2013. This article is published with open access at Springerlink.com

\begin{abstract}
A generalized Cauchy problem for nonlinear hyperbolic functional differential systems is considered. A theorem on the existence of weak solutions is proved. The initial problem is transformed into a system of functional integral equations for an unknown function and for their partial derivatives with respect to spatial variables. The existence of solutions of this system is proved by using a method of successive approximations. It is shown a result on the differentiability of solutions with respect to initial functions. This is the main result of the paper.
\end{abstract}

Keywords Functional differential equations - Generalized Cauchy problem . Volterra condition · Differentiability of solutions

Mathematics Subject Classification (2010) $\quad 35 \mathrm{R} 10 \cdot 35 \mathrm{~F} 25 \cdot 35 \mathrm{~A} 01$

\section{Introduction}

For any metric spaces $U$ and $V$ we denote by $C(U, V)$ the class of all continuous functions from $U$ into $V$. We will use vectorial inequalities if the same inequalities hold between corresponding components. Suppose that $M \in C\left([0, a], \mathbb{R}_{+}^{n}\right), a>0, \mathbb{R}_{+}=[0,+\infty), M$ is nondecreasing and $M(0)=0_{[n]}$ where $0_{[n]}=(0, \ldots, 0) \in \mathbb{R}^{n}$. Let $E$ be the Haar pyramid

$$
E=\left\{(t, x) \in \mathbb{R}^{1+n}: t \in[0, a],-b+M(t) \leq x \leq b-M(t)\right\},
$$

where $b \in \mathbb{R}^{n}$ and $b>M(a)$. Suppose that $b_{0} \in R_{+}$and $M^{-}, M^{+} \in C\left(\left[-b_{0}, 0\right], R^{n}\right)$, $M^{-}=\left(M_{1}^{-}, \ldots, M_{n}^{-}\right), M^{+}=\left(M_{1}^{+}, \ldots, M_{n}^{+}\right)$and $M^{-}(0)=-b, M^{+}(0)=b$ and

Corresponding Author: Prof. dr hab. Zdzislaw Kamont (1942-2012) passed away suddenly on September 3, 2012.

Z. Kamont $(\bowtie)$

Institute of Mathematics, University of Gdańsk, Wit Stwosz Street 57,

80-952 Gdańsk, Poland 
$M^{-}(t)<M^{+}(t)$ for $t \in\left[-b_{0}, 0\right]$. Set $E_{0}=\left\{(t, x) \in \mathbb{R}^{1+n}: t \in\left[-b_{0}, 0\right], M^{-}(t) \leq\right.$ $\left.x \leq M^{+}(t)\right\}$. For $(t, x) \in E$ we define

$$
D[t, x]=\left\{(\tau, y) \in \mathbb{R}^{1+n}: \tau \leq 0,(t+\tau, x+y) \in E_{0} \cup E\right\} .
$$

Then $D[t, x]=D_{0}[t, x] \cup D_{\star}[t, x)$ where

$$
\begin{aligned}
& D_{0}[t, x]=\left\{(\tau, y) \in \mathbb{R}^{1+n}:-b_{0}-t \leq \tau \leq-t,-x+M^{-}(t+\tau) \leq y \leq-x\right. \\
& \left.\quad+M^{+}(t+\tau)\right\}, \\
& D_{\star}[t, x]=\{(\tau, y):-t \leq \tau \leq 0,-b-x+M(\tau+t) \leq y \leq b-x-M(\tau+t)\} .
\end{aligned}
$$

Set $b^{-}=\left(b_{1}^{-}, \ldots, b_{n}^{-}\right), b^{+}=\left(b_{1}^{+}, \ldots, b_{n}^{+}\right)$where

$$
b_{i}^{-}=\min \left\{M_{i}^{-}(t): t \in\left[-b_{0}, 0\right]\right\}, \quad b_{i}^{+}=\max \left\{M_{i}^{+}(t): t \in\left[-b_{0}, 0\right]\right\}, \quad 1 \leq i \leq n,
$$

and $B=\left[-b_{0}-a, 0\right] \times\left[-b+b^{-}, b+b^{+}\right]$. Then $D[t, x] \subset B$ for $(t, x) \in E$. Denote by $\mathbb{N}$ the set of natural numbers. Let $\mathbb{S}$ be the class of all sequences $p=\left\{p_{k}\right\}_{k \in \mathbb{N}}$ where $p_{k} \in \mathbb{R}$ for $k \in \mathbb{N}$. Write

$$
E_{0 . k}=\left(E_{0} \cup E\right) \cap\left(\left[-b_{0}, a_{k}\right] \times \mathbb{R}^{n}\right), \quad k \in \mathbb{N},
$$

where $a_{k} \geq 0$ for $k \in \mathbb{N}$ and $\kappa=\sup \left\{a_{k}: k \in \mathbb{N}\right\}<a$. For a function $z: E_{0} \cup E \rightarrow \mathbb{S}$, $z=\left\{z_{k}\right\}_{k \in \mathbb{N}}$, and for a point $(t, x) \in E$ we define $z_{(t, x)}: D[t, x] \rightarrow \mathbb{S}, z_{(t, x)}=\left\{\left(z_{k}\right)_{(t, x)}\right\}_{k \in \mathbb{N}}$, by

$$
\left(z_{k}\right)_{(t, x)}(\tau, y)=z_{k}(t+\tau, x+y), \quad(\tau, y) \in D[t, x], k \in \mathbb{N} .
$$

Then $z_{(t, x)}$ is the restriction of $z$ to the set $\left(E_{0} \cup E\right) \cap\left(\left[-b_{0}, t\right] \times \mathbb{R}^{n}\right)$ and this restriction is shifted to the set $D[t, x]$.

Suppose the $\phi_{0}:[0, a] \rightarrow \mathbb{R}$ and $\phi: E \rightarrow \mathbb{R}^{n}, \phi=\left(\phi_{1}, \ldots, \phi_{n}\right)$ are given functions. The requirements on $\phi_{0}$ and $\phi$ are that $0 \leq \phi_{0}(t) \leq t$ and $\left(\phi_{0}(t), \phi(t, x)\right) \in E$ for $(t, x) \in E$. Write $\varphi(t, x)=\left(\phi_{0}(t), \phi(t, x)\right)$ on $E$. Let $l^{\infty}$ be the class of all sequences $p=\left\{p_{k}\right\}_{k \in \mathbb{N}}$ such that $\|p\|_{\infty}=\sup \left\{\left|p_{k}\right|: k \in \mathbb{N}\right\}<\infty$. Set $\Omega=E \times C\left(B, l^{\infty}\right) \times C\left(B, l^{\infty}\right) \times \mathbb{R}^{n}$ and suppose that

$$
F: \Omega \rightarrow \mathbb{S}, F=\left\{F_{k}\right\}_{k \in \mathbb{N}} \text {, and } \psi=\left\{\psi_{k}\right\}_{k \in \mathbb{N}}, \psi_{k}: E_{0 . k} \rightarrow \mathbb{R} \text { for } k \in \mathbb{N},
$$

are given functions. We will say that $F$ satisfies condition $(V)$ if for each $(t, x, q) \in E \times \mathbb{R}^{n}$ and for $v, \tilde{v}, w, \tilde{w} \in C\left(B, l^{\infty}\right)$ such that $v(\tau, y)=\tilde{v}(\tau, y)$ for $(\tau, y) \in D[t, x]$ and $w(\tau, y)=$ $\bar{w}(\tau, y)$ for $(\tau, y) \in D[\varphi(t, x)]$ we have $F(t, x, v, w, q)=F(t, x, \tilde{v}, \tilde{w}, q)$. Note that the condition $(V)$ means that the values of $F$ at the point $(t, x, v, w, q) \in \Omega$ depends on $(t, x, q)$ and on the restriction of $v$ and $w$ to the sets $D[t, x]$ and $D[\varphi(t, x)]$ only.

Let us denote by $z=\left\{z_{k}\right\}_{k \in \mathbb{N}}$ an unknown function of the variables $(t, x), x=$ $\left(x_{1}, \ldots, x_{n}\right)$. We consider the system of functional differential equations

$$
\partial_{t} z_{k}(t, x)=F_{k}\left(t, x, z_{(t, x)}, z_{\varphi(t, x)}, \partial_{x} z_{k}(t, x)\right), \quad k \in \mathbb{N},
$$

with the initial conditions

$$
z_{k}(t, x)=\psi_{k}(t, x) \text { on } E_{0 . k}, \quad k \in \mathbb{N},
$$

where $\partial_{x} z_{k}=\left(\partial_{x_{1}} z_{k}, \ldots, \partial_{x_{n}} z_{k}\right), 1 \leq i \leq k$. We assume that $F$ satisfies the condition $(V)$.

Write

$$
\begin{aligned}
& E_{t}=\left(E_{0} \cup E\right) \cap\left(\left[-b_{0}, t\right] \times \mathbb{R}^{n}\right), \quad S_{t}=[-b+M(t), b-M(t)], \quad t \in[0, a], \\
& I_{c . k}[x]=\left\{t \in\left[a_{k}, c\right]:-b+M(t) \leq x \leq b-M(t)\right\}, \quad x \in[-b, b], k \in \mathbb{N},
\end{aligned}
$$


where $\kappa<c \leq a$. We consider weak solutions of initial problems. A function $\tilde{z}: E_{c} \rightarrow \mathbb{S}$, $\tilde{z}=\left\{\tilde{z}_{k}\right\}_{k \in \mathbb{N}}$, where $\kappa<c \leq a$, is a solution to (1), (2) provides

(i) $\tilde{z}_{(t, x)} \in C\left(B, l^{\infty}\right)$ for $(t, x) \in E_{c}, 0 \leq t \leq c$ and $\partial_{x} \tilde{z}_{k}$ exist and they are continuous on $E \cap\left(\left[a_{k}, c\right] \times \mathbb{R}^{n}\right)$ for $k \in \mathbb{N}$,

(ii) for each $k \in \mathbb{N}$ and $x \in[-b, b]$ the function $\tilde{z}_{k}(\cdot, x): I_{c . k}[x] \rightarrow \mathbb{R}$ is absolutely continuous,

(iii) for each $x \in[-b, b]$ and for $k \in \mathbb{N}$, the $k$-th equation in (1) is satisfied for almost all $t \in I_{c . k}[x]$ and conditions (2) hold.

System (1) with initial conditions (2) is called a generalized Cauchy problem. If $a_{k}=0$ for $k \in \mathbb{N}$ then (1), (2) reduces to the classical Cauchy problem. The following question is considered in the paper. We prove that under natural assumptions on given functions there exists exactly one solution to (1), (2) defined on $E_{c}$ and we give an estimate of $c \in(\kappa, a]$. Let us denote by $\mathbb{X}$ the class of all $\psi=\left\{\psi_{k}\right\}_{k \in \mathbb{N}}, \psi_{k}: E_{0 . k} \rightarrow \mathbb{R}$ for $k \in \mathbb{N}$, such that there exists exactly one solution $\Xi[\psi]: E_{c} \rightarrow l^{\infty}$ to (1), (2). We give a construction of the space $\mathbb{X}$. We prove that there is $\mathbb{Y} \subset \mathbb{X}$ such that for each $\psi \in \mathbb{Y}$ there exists the Fréchet derivative $\partial \Xi[\psi]$ of $\Xi$ at $\psi$. Moreover, if $\psi \in \mathbb{Y}$ and $\pi \in \mathbb{X}$ and $\bar{z}=\partial \Xi[\psi] \pi$ then $\bar{z}$ is a solution of an integral functional system generated by (1), (2).

Until now there have not been any results on the differentiability with respect to initial functions for solutions of nonlinear hyperbolic functional differential systems. Our theorems are new also in the case when (1), (2) reduces to a finite functional differential system.

In recent years, a number of papers concerning first order partial functional differential equations have been published. The following questions were considered: functional differential inequalities generated by initial or mixed problems and their applications $[1,5,6,12]$, existence theory of classical or weak solutions of equations or finite systems with initial or initial boundary conditions [2-4,9,14,22] approximate solutions of functional differential problems [15-17,25]. Essential extensions of some ideas concerning generalized solution of Hamilton-Jacobi equations are given in $[20,21]$ where viscosity solutions are considered.

Infinite systems of first order partial functional differential equations were first treated in $[18,19]$. The existence result presented in [18] is based on a method of successive approximations which was introduced by Ważewski [23] for systems without the functional dependence. Existence results for initial problems [11] and for mixed problems [8] related to infinite systems of nonlinear equations are obtained by a quasilinearization procedure and by construction of functional integral systems for unknown functions and for their derivatives with respect to spatial variables. This method was initiated in [7] for nonlinear systems without functional variables. Differential inequalities and suitable comparison results for infinite systems of hyperbolic functional differential inequalities are given in [13,19].

Information on applications of functional differential equations can be found in [12,24]. The monograph [10] contains results on differentiability with respect to initial functions for solutions of ordinary functional differential equations.

The paper is organized as follows. In Sect. 2 we transform the generalized Cauchy problem into a system of integral functional equations. This system is solved in Sect. 3 by the method of successive approximations. As a consequence we obtain a theorem on the existence of solution to (1), (2) an on continuous dependence of solutions on initial functions.

A theorem on the differentiability of solutions with respect to initial functions is presented in Sect. 4. It is the main result of the paper.

Two types of assumptions are needed in theorems on the existence of solutions to initial or initial boundary value problems related to hyperbolic functional differential systems. The first type conditions concern the bicharacteristics. The second type assumptions concern the 
regularity of given functions. The authors of the papers $[2-4,8,9,11,18,22]$ have assumed that the partial derivatives of given functions satisfy the Lipschitz condition with respect to all variables except for $t$. These conditions are global. Our assumptions on the regularity of given functions are more general. We assume that the partial derivatives of $F$ satisfy the Lipschitz condition and suitable estimates are local with respect to all variables. It is clear that there are differential systems with deviated variables and differential integral systems such that local estimates hold and global inequalities are not satisfied.

Motivations for investigations of functional differential systems with two functional variables are given in Remark 2.4. We give examples of functional differential systems which can be derived from (1) be specializing the operator $F$.

Example 1.1 Suppose that $G: E \times l^{\infty} \times l^{\infty} \times \mathbb{R}^{n} \rightarrow \mathbb{S}, G=\left\{G_{k}\right\}_{k \in \mathbb{N}}$, is a given function and $F$ is defined by

$$
F(t, x, v, w, q)=G\left(t, x, v\left(0,0_{[n]}\right), w\left(0,0_{[n]}\right), q\right) \text { on } \Omega .
$$

Then (1) reduces to the system of differential equations with deviated variables

$$
\partial_{t} z_{k}(t, x)=G_{k}\left(t, x, z(t, x), z(\varphi(t, x)), \partial_{x} z_{k}(t, x)\right), \quad k \in \mathbb{N} .
$$

Example 1.2 Suppose that $b_{0}>0$ and that there is $\bar{M} \in \mathbb{R}_{+}^{n}$ such that $M(t)=\bar{M} t$ for $t \in[0, a]$. Then $E$ is the classical Haar pyramid

$$
E=\left\{(t, x) \in \mathbb{R}^{1+n}: t \in[0, a],-b+\bar{M} t \leq x \leq b-\bar{M} t\right\} .
$$

Set $M^{-}(t)=-b+\bar{M} t$ and $M^{+}(t)=b-\bar{M} t$ for $t \in\left[-b_{0}, 0\right]$. Then

$$
E_{0}=\left\{(t, x) \in \mathbb{R}^{1+n}: t \in\left[-b_{0}, 0\right],-b+\bar{M} t \leq x \leq b-\bar{M} t\right\} .
$$

Suppose that $0<v<\mu \leq b_{0}$ and $0_{[n]}<h \leq \bar{M} v$. For the above $G$ we put

$$
F(t, x, v, w, q)=G\left(t, x, \int_{-h}^{h} v(-\mu, y) d y, w\left(0,0_{[n]}\right), q\right) \quad \text { on } \Omega .
$$

Then (1) reduces to the differential integral system

$$
\partial_{t} z_{k}(t, x)=G\left(t, x, \int_{x-h}^{x+h} z(t-\mu, y) d y, z(\varphi(t, x)), \partial_{x} z_{k}(t, x)\right), \quad k \in \mathbb{N} .
$$

It is clear that more complicated examples of differential functional systems can be derived from (1).

\section{Integral functional equations}

Let $\mathbb{L}\left([\tau, t], \mathbb{R}_{+}^{n}\right),[\tau, t] \subset \mathbb{R}$, be the class of all $\omega:[\tau, t] \rightarrow \mathbb{R}_{+}^{n}$ which are integrable on $[\tau, t]$. For $x \in \mathbb{R}^{n}, x=\left(x_{1}, \ldots, x_{n}\right)$, we put $\|x\|=\left|x_{1}\right|+\ldots+\left|x_{n}\right|$. We use the symbol "o" to denote the scalar product in $\mathbb{R}^{n}$. We denote by $M_{n \times n}$ be the class of all $n \times n$ matrices with real elements. For $A \in M_{n \times n}$ where $A=\left[a_{i j}\right]_{i, j=1, \ldots, n}$ we put

$$
\|A\|_{n \times n}=\max \left\{\sum_{j=1}^{n}\left|a_{i j}\right|: 1 \leq i \leq n\right\} .
$$


Let $M_{\infty \times n}$ be the class of all real matrices $B=\left[b_{i j}\right]_{i \in \mathbb{N}, 1 \leq j \leq n}$ with the finite norm

$$
\|B\|_{\infty \times n}=\sup \left\{\sum_{j=1}^{n}\left|b_{i j}\right|: i \in \mathbb{N}\right\} .
$$

We will use the symbol $M_{\infty \times \infty}$ to denote the set of real matrices $C=\left[c_{i j}\right]_{i, j \in \mathbb{N}}$ with the finite norm:

$$
\|C\|_{\infty \times \infty}=\sup \left\{\sum_{j=1}^{\infty}\left|c_{i j}\right|: i \in \mathbb{N}\right\} .
$$

For the above $B \in M_{\infty \times n}$ and $C \in M_{\infty \times \infty}$ we write $b_{[i]}=\left(b_{i 1}, \ldots, b_{i n}\right)$, and $c_{[i]}=$ $\left(c_{i 1}, c_{i 2}, \ldots\right), i \in \mathbb{N}$.

We will denote by $C L(B, \mathbb{R})$ the class of all linear and continuous operators defined on $C(B, \mathbb{R})$ and taking values in $\mathbb{R}$. The norm in the space $C L(B, \mathbb{R})$ generated by the maximum norm in $C(B, \mathbb{R})$ will be denoted by $\|\cdot\|_{\star}$. Let $C L\left(B, M_{\infty \times \infty}\right)$ be the class of all $\Xi=\left[\Xi_{i j}\right]_{i, j \in \mathbb{N}}$ such that

$$
\|\Xi\|_{\infty \times \infty ; \star}=\sup \left\{\left\|\Xi_{[i]}\right\|_{\infty ; \star}: i \in \mathbb{N}\right\}<+\infty,
$$

where

$$
\left\|\Xi_{[i]}\right\|_{\infty ; \star}=\sum_{j=1}^{\infty}\left\|\Xi_{i j}\right\|_{\star}, \quad \Xi_{[i]}=\left(\Xi_{i 1}, \Xi_{i 2}, \ldots\right) \quad \text { for } \quad i \in \mathbb{N}
$$

Now we define some function spaces. Given $\bar{c}=\left(c_{0}, c_{1}, c_{2}\right) \in \mathbb{R}_{+}^{3}$, we denote by $\mathbb{X}$ the set of all $\psi=\left\{\psi_{k}\right\}_{k \in \mathbb{N}}$ such that for each $k \in \mathbb{N}$ we have

(i) $\psi_{k} \in C\left(E_{0 . k}, \mathbb{R}\right)$, the derivatives $\partial_{x} \psi_{k}=\left(\partial_{x_{1}} \psi_{k}, \ldots, \partial_{x_{n}} \psi_{k}\right)$ exist on $E_{0 . k}$ and $\partial_{x} \psi_{k} \in$ $C\left(E_{0 . k}, \mathbb{R}^{n}\right)$,

(ii) the estimates

$$
\begin{aligned}
& \left|\psi_{k}(t, x)\right| \leq c_{0}, \quad\left\|\partial_{x} \psi_{k}(t, x)\right\| \leq c_{1}, \\
& \left\|\partial_{x} \psi_{k}(t, x)-\partial_{x} \psi_{k}(t, \bar{x})\right\| \leq c_{2}\|x-\bar{x}\|
\end{aligned}
$$

are satisfied on $E_{0 . k}$.

Let $\psi \in \mathbb{X}, \psi=\left\{\psi_{k}\right\}_{k \in \mathbb{N}}$, be given and $\kappa<c \leq a$. We denote by $C_{\psi \cdot c}$ the class of all $z \in C\left(E_{c}, l^{\infty}\right), z=\left\{z_{k}\right\}_{k \in \mathbb{N}}$, such that $z_{k}(t, x)=\psi_{k}(t, x)$ on $E_{0 . k}$ for $k \in \mathbb{N}$. For the above $\psi$ and $\kappa<c \leq a$ we denote by $C_{\partial \psi_{k} . c}, k \in \mathbb{N}$, the class of all $\vartheta \in C\left(E_{c}, \mathbb{R}^{n}\right)$ such that $\vartheta(t, x)=\partial_{x} \psi_{k}(t, x)$ on $E_{0 . k}$.

Write $\Omega_{I}=[-b, b] \times C\left(B, l^{\infty}\right) \times C\left(B, l^{\infty}\right) \times \mathbb{R}^{n}$ and $\Omega_{t}=S_{t} \times C\left(B, l^{\infty}\right) \times C\left(B, l^{\infty}\right) \times$ $\mathbb{R}^{n}, t \in[0, a]$.

Assumption $H_{0}[F]$. The function $F: \Omega \rightarrow \mathbb{S}$ satisfies the condition (V) and

1. for each $(x, v, w, q) \in \Omega_{I}$ the function $F:(\cdot, x, v, w, q): I[x] \rightarrow \mathbb{S}$ is measurable and there is $\alpha \in \mathbb{L}\left([0, a], \mathbb{R}_{+}\right)$such that

$$
\left\|F\left(t, x, \theta, \theta, 0_{[n]}\right)\right\|_{\infty} \leq \alpha(t) \quad \text { on } E
$$

where $\theta \in C\left(B, l^{\infty}\right)$ is given by $\theta(\tau, s)=0_{l^{\infty}}$ and $0_{l^{\infty}}$ is the zero in the space $l^{\infty}$, 
2. for each $P=(t, x, v, w, q) \in \Omega$ there exist the derivatives

$$
\partial_{x} F(P)=\left[\partial_{x_{j}} F_{i}(P)\right]_{i \in \mathbb{N}, 1 \leq j \leq n}, \quad \partial_{q} F(P)=\left[\partial_{q_{j}} F_{i}(P)\right]_{i \in \mathbb{N}, 1 \leq j \leq n},
$$

and the functions $\partial_{x} F(\cdot, x, v, w, q), \partial_{q} F(\cdot, x, v, w, q): I[x] \rightarrow M_{\infty \times n}$ are measurable and the functions $\partial_{x} F(t, \cdot), \partial_{q} F(t, \cdot): \Omega_{t} \rightarrow M_{\infty \times n}$ are continuous,

3. for each $P=(t, x, v, w, q) \in \Omega$ there exist the Frechét derivatives

$$
\partial_{v} F(P)=\left[\partial_{v_{j}} F_{i}(P)\right]_{i, j \in \mathbb{N}}, \quad \partial_{w} F(P)=\left[\partial_{w_{j}} F_{i}(P)\right]_{i, j \in \mathbb{N}},
$$

and for each $\tilde{w} \in C(B, \mathbb{R})$ we have

$$
\partial_{v} F(\cdot, x, v, w, q) \tilde{w}, \partial_{q} F(\cdot, x, v, w, q) \tilde{w}: I[x] \rightarrow M_{\infty \times \infty} \text { are measurable }
$$

and

$$
\partial_{v} F(t, \cdot) \tilde{w}, \partial_{w} F(t, \cdot) \tilde{w}: \Omega_{I} \rightarrow M_{\infty \times \infty} \text { are continuous }
$$

where

$$
\partial_{v} F(P) \tilde{w}=\left[\partial_{v_{j}} F_{i}(P) \tilde{w}\right]_{i, j \in \mathbb{N}}, \quad \partial_{w} F(P) \tilde{w}=\left[\partial_{w_{j}} F_{i}(P) \tilde{w}\right]_{i, j \in \mathbb{N}},
$$

4. there are $\beta \in \mathbb{L}\left([0, a], \mathbb{R}_{+}\right)$and $L \in \mathbb{L}\left([0, a], \mathbb{R}_{+}^{n}\right), L=\left(L_{1}, \ldots, L_{n}\right)$, such that for $P=(t, x, v, w, q) \in \Omega$ we have

$$
\left\|\partial_{x} F(P)\right\|_{\infty \times n} \leq \beta(t), \quad\left\|\partial_{v} F(P)\right\|_{\infty \times \infty ; \star} \leq \beta(t), \quad\left\|\partial_{w} F(P)\right\|_{\infty \times \infty ; \star} \leq \beta(t),
$$

and

$$
\left(\left|\partial_{q_{1}} F_{k}(P)\right|, \ldots,\left|\partial_{q_{n}} F_{k}(P)\right|\right) \leq L(t), \quad k \in \mathbb{N},
$$

and for $t \in[0, a]$ we have

$$
M(t)=\int_{0}^{t} L(\xi) d \xi .
$$

Assumption $H[\varphi]$. The functions $\phi_{0}:[0, a] \rightarrow \mathbb{R}, \phi: E \rightarrow \mathbb{R}^{n}, \phi=\left(\phi_{1}, \ldots, \phi_{n}\right)$, are continuous and

1. $0 \leq \phi_{0} \leq t$ for $t \in[0, a]$ and $\varphi(t, x)=\left(\phi_{0}(t), \phi(t, x)\right) \in E$,

2. there exist the derivatives

$$
\partial_{x} \phi(t, x)=\left[\partial_{x_{j}} \phi_{i}(t, x)\right]_{i, j=1, \ldots, n}
$$

and $\partial_{x} \phi \in C\left(E, M_{n \times n}\right)$,

3. the constant $Q_{0} \in \mathbb{R}_{+}$is defined by the relation $\left\|\partial_{x} \phi(t, x)\right\|_{n \times n} \leq Q_{0}$ for $(t, x) \in E$ and there is $Q \in \mathbb{R}_{+}$such that

$$
\left\|\partial_{x} \phi(t, x)-\partial_{x} \phi(t, \bar{x})\right\|_{n \times n} \leq Q \text { on } E .
$$

Suppose that Assumptions $H_{0}[F], H[\varphi]$ are satisfied and $\psi \in \mathbb{X}, z \in C_{\psi . c}, u \in$ $C\left(E_{c}, M_{\infty \times \infty}\right)$ where $\kappa<c \leq a$ and

$$
\begin{aligned}
& z=\left\{z_{k}\right\}_{k \in \mathbb{N}}, \quad u=\left[u_{i j}\right]_{i \in \mathbb{N}, 1 \leq j \leq n}, \\
& u_{[i]}=\left(u_{i 1}, \ldots, u_{i n}\right), \quad u_{[i]} \in C_{\partial \psi_{i} . c} \quad \text { for } i \in \mathbb{N} .
\end{aligned}
$$


Write $S\left[z, u_{[k]}\right](\tau, x)=\left(\tau, x, z_{(\tau, x)}, z_{\varphi(t, x)}, u_{[k]}(\tau, x)\right), k \in \mathbb{N}$. We consider the Cauchy problem

$$
\omega^{\prime}(\tau)=-\partial_{q} F_{k}\left(S\left[z, u_{[k]}\right](\tau, \omega(\tau))\right), \quad \omega(t)=x,
$$

where $(t, x) \in E, a_{k} \leq t \leq a$ and $\partial_{q} F_{k}=\left(\partial_{q_{1}} F_{k}, \ldots, \partial_{q_{n}} F_{k}\right)$. Let us denote by $g_{[k]}\left[z, u_{[k]}\right](\cdot, t, x)$ the solution of $(9)$. The function $g_{[k]}\left[z, u_{[k]}\right](\cdot, t, x)$ is the $k$-th bicharactersitic of (1) corresponding to $(z, u)$. Write

$$
\begin{aligned}
& u_{(t, x)}=\left[\left(u_{i j}\right)_{(t, x)}\right]_{i \in \mathbb{N}, 1 \leq j \leq n}, \\
& \left(u_{[k]}\right)_{(t, x)}=\left(\left(u_{k 1}\right)_{(t, x)}, \ldots,\left(u_{k n}\right)_{(t, x)}\right), \quad k \in \mathbb{N},
\end{aligned}
$$

and $P\left[z, u_{[k]}\right](\tau, t, x)=S\left[z, u_{[k]}\right]\left(\tau, g_{[k]}\left[z, u_{[k]}\right](\tau, t, x)\right), k \in \mathbb{N}$. For $P \in \Omega$ and for $\tilde{w} \in C\left(B, l^{\infty}\right), \quad \tilde{w}=\left\{\tilde{w}_{k}\right\}_{k \in \mathbb{N}}, \quad \tilde{W} \in C\left(B, M_{\infty \times n}\right), \quad \tilde{W}=\left[\tilde{w}_{i j}\right]_{i \in \mathbb{N}, 1 \leq j \leq n}$, we put

$$
\begin{aligned}
\partial_{v} F_{k}(p) \diamond \tilde{w} & =\sum_{j=1}^{\infty} \partial_{v_{j}} F_{k}(P) \tilde{w}_{j}, \\
\partial_{v} F_{k}(P) \star \tilde{W} & =\left[\sum_{j=1}^{\infty} \partial_{v_{j}} F_{k}(P) \tilde{w}_{j 1}, \ldots, \sum_{j=1}^{\infty} \partial_{v_{j}} F_{k}(P) \tilde{w}_{j n}\right],
\end{aligned}
$$

where $k \in \mathbb{N}$. In similar way we define the expressions $\partial_{w} F_{k}(P) \diamond \tilde{w}, \partial_{w} F_{k}(P) \star \tilde{W}$ for $k \in \mathbb{N}$. Let us denote by $\mathbb{F}[z, u]=\left\{\mathbb{F}_{k}[z, u]\right\}_{k \in \mathbb{N}}$ the function defined in the following way:

$$
\mathbb{F}_{k}[z, u](t, x)=\psi_{k}(t, x) \text { on } E_{0 . k}
$$

and

$$
\begin{aligned}
& \mathbb{F}_{k}[z, u](t, x)=\psi_{k}\left(a_{k}, g_{[k]}\left[z, u_{[k]}\right]\left(a_{k}, t, x\right)\right)+\int_{a_{k}}^{t} F_{k}\left(P\left[z, u_{[k]}\right](\tau, t, x)\right) d \tau \\
& \quad-\int_{a_{k}}^{t} \partial_{q} F_{k}\left(P\left[z, u_{[k]}\right](\tau, t, x)\right) \diamond u_{[k]}\left(\tau, g_{[k]}\left[z, u_{[k]}\right](\tau, t, x)\right) d \tau \quad \text { on } E \cap\left(\left[a_{k}, c\right] \times \mathbb{R}^{n}\right) .
\end{aligned}
$$

Moreover we put

$$
\begin{aligned}
& \mathbb{G}[z, u]=\left\{\mathbb{G}_{i j}[z, u]\right\}_{i \in \mathbb{N}, 1 \leq j \leq n}, \\
& \mathbb{G}_{[k]}[z, u]=\left(\mathbb{G}_{k 1}[z, u], \ldots, \mathbb{G}_{k n}[z, u]\right), \quad k \in \mathbb{N},
\end{aligned}
$$

where

$$
\mathbb{G}_{[k]}[z, u](t, x)=\partial_{x} \psi_{k}(t, x) \quad \text { on } E_{0 . k}
$$

and

$$
\mathbb{G}_{[k]}[z, u](t, x)=\partial_{x} \psi_{k}\left(a_{k}, g_{[k]}\left[z, u_{[k]}\right]\left(a_{k}, t, x\right)\right)+\int_{a_{k}}^{t} \partial_{x} F_{k}\left(P\left[z, u_{[k]}\right](\tau, t, x)\right) d \tau
$$


$+\int_{a_{k}}^{t} \partial_{v} F_{k}\left(P\left[z, u_{[k]}\right](\tau, t, x)\right) \star u_{\left(\tau, g_{[k]}\left[z, u_{[k]}\right](\tau, t, x)\right)} d \tau$
$+\int_{a_{k}}^{t} \partial_{w} F_{k}\left(P\left[z, u_{[k]}\right](\tau, t, x)\right) \star\left[u_{\varphi\left(\tau, g_{[k]}\left[z, u_{[k]}\right](\tau, t, x)\right)} \partial_{x} \phi\left(\tau, g_{[k]}\left[z, u_{[k]}\right](\tau, t, x)\right)\right] d \tau$

on $E \cap\left(\left[a_{k}, c\right] \times \mathbb{R}^{n}\right)$. The functions $u_{\varphi(\tau, y)} \partial_{x} \phi(\tau, y): B \rightarrow M_{\infty \times n}, y=g_{[k]}\left[z, u_{[k]}\right](\tau, t, s)$, are defined by

$$
u_{\varphi(\tau, y)} \partial_{x} \phi(\tau, y)=\left[\sum_{\mu=1}^{n}\left(u_{i \mu}\right)_{\varphi(\tau, y)} \partial_{x_{j}} \phi_{\mu}(\tau, y)\right]_{i \in \mathbb{N}, 1 \leq j \leq n} .
$$

We consider the system of functional integral equations

$$
z=\mathbb{F}[z, u], \quad u=\mathbb{G}[z, u] .
$$

We show that under natural assumptions on given functions there exists a solution $(\bar{z}, \bar{u}): E_{c} \rightarrow l^{\infty} \times M_{\infty \times n}$ of $(10)$ and there exist the derivatives $\partial_{x} \bar{z}_{k}=\left(\partial_{x_{1}} \bar{z}_{k}, \ldots, \partial_{x_{n}} \bar{z}_{k}\right)$, $k \in \mathbb{N}$, and $\bar{u}_{[k]}=\partial_{x} \bar{z}_{k}$ for $k \in \mathbb{N}$.

We first give estimates of solutions to (10). For $z \in C\left(E_{c}, l^{\infty}\right), \vartheta \in C\left(E_{c}, \mathbb{R}^{n}\right), u \in$ $C\left(E_{c}, M_{\infty \times n}\right)$ we define the seminorms

$$
\begin{aligned}
& \|z\|_{\left(t, l^{\infty}\right)}=\max \left\{\|z(\tau, s)\|_{\infty}:(\tau, s) \in E_{t}\right\}, \\
& \|\vartheta\|_{\left(t, \mathbb{R}^{n}\right)}=\max \left\{\|\vartheta(\tau, s)\|_{\infty}:(\tau, s) \in E_{t}\right\}, \\
& \|v\|_{\left(t, M_{\infty \times n}\right)}=\max \left\{\|u(\tau, s)\|_{\infty \times n}:(\tau, s) \in E_{t}\right\},
\end{aligned}
$$

where $t \in[0, c]$.

Lemma 2.1 Suppose that Assumptions $H_{0}[F], H[\varphi]$ are satisfied and

1. $\psi \in \mathbb{X}$ and $\kappa<c \leq a$,

2. the functions $\bar{z}: E_{c} \rightarrow l^{\infty}, \bar{u}: E_{c} \rightarrow M_{\infty \times n}$ satisfy (10) and $\bar{z} \in C\left(E_{c}, l^{\infty}\right), \bar{u} \in$ $C\left(E_{c}, M_{\infty \times n}\right)$.

Then

$$
\|\bar{z}\|_{\left(t, l^{\infty}\right)} \leq \zeta(t), \quad\|\bar{u}\|_{\left(t, M_{\infty \times n}\right)} \leq \chi(t) \quad \text { for } t \in[0, c],
$$

where

$$
\begin{aligned}
& \chi(t)=c_{1} \exp \left\{\left(1+Q_{0}\right) \int_{0}^{t} \beta(\xi) d \xi\right\}+\int_{0}^{t} \beta(\xi) \exp \left\{\left(1+Q_{0}\right) \int_{\xi}^{t} \beta(\tau) d \tau\right\} d \xi, \\
& \zeta(t)=c_{0} \exp \left\{2 \int_{0}^{t} \beta(\xi) d \xi\right\}+\int_{0}^{t} \bar{\gamma}(\xi) \exp \left\{2 \int_{\xi}^{t} \beta(\tau) d \tau\right\} d \xi, \\
& \bar{\gamma}(t)=\alpha(t)+(\beta(t)+\|L(t)\|) \chi(t) .
\end{aligned}
$$


Proof Write $\bar{\zeta}(t)=\|\bar{z}\|_{\left(t, l^{\infty}\right)}, \bar{\chi}(t)=\|\bar{u}\|_{\left(t, M_{\infty \times n}\right)}, t \in[0, c]$. It follows from Assumptions $H_{0}[F]$ and $H[\varphi]$ that the $(\bar{\zeta}, \bar{\chi})$ satisfy the integral inequalities

$$
\begin{aligned}
& \bar{\zeta}(t) \leq c_{0}+\int_{0}^{t} \alpha(\xi) d \xi+2 \int_{0}^{t} \beta(\xi) \bar{\zeta}(\xi) d \xi+\int_{0}^{t}(\beta(\xi)+\|L(\xi)\|) \bar{\chi}(\xi) d \xi, \\
& \bar{\chi}(t) \leq c_{1}+\int_{0}^{t} \beta(\xi) d \xi+\left(1+Q_{0}\right) \int_{0}^{t} \beta(\xi) \bar{\chi}(\xi) d \xi, \quad t \in[0, c] .
\end{aligned}
$$

The functions $(\zeta, \chi)$ satisfy the integral equations corresponding to the above inequalities. This proves the lemma.

Suppose that $\psi \in \mathbb{X}, \kappa<c \leq a$ and $d_{0}, r_{0} \in \mathbb{R}_{+}$and $d_{0} \geq c_{1}, r_{0} \geq c_{2}$. We denote by $C_{\psi . c}\left[\zeta, d_{0}\right]$ the class of all $z \in C_{\psi . c}$ such that

$$
\|z\|_{\left(t, l^{\infty}\right)} \leq \zeta(t) \text { for } t \in[0, a]
$$

and

$$
\left|z_{k}(t, x)-z_{k}(t, \bar{x})\right| \leq d_{0}\|x-\bar{x}\| \quad \text { for }(t, x),(t, \bar{x}) \in E \cap\left(\left[a_{k}, a\right] \times \mathbb{R}^{n}\right), k \in \mathbb{N} .
$$

Let $C_{\partial \psi_{k} . c}\left[\chi, r_{0}\right], k \in \mathbb{N}$, be the class of all $\vartheta \in C_{\partial \psi_{k} . c}$ satisfying the conditions:

$$
\|\vartheta\|_{\left(t, \mathbb{R}^{n}\right)} \leq \chi(t) \text { for } t \in\left[a_{k}, c\right]
$$

and

$$
\|\vartheta(t, x)-\vartheta(t, \bar{x})\| \leq r_{0}\|x-\bar{x}\| \quad \text { on } E \cap\left(\left[a_{k}, c\right] \times \mathbb{R}^{n}\right) .
$$

Write $d=\zeta(a), r=\chi(a)$ and $\Omega[d, r]=E \times K_{C\left(B, l^{\infty}\right)}[d] \times K_{C\left(B, l^{\infty}\right)}[d] \times K_{\mathbb{R}^{n}}[r]$ where $K_{C\left(B, l^{\infty}\right)}[d]=\left\{w \in C\left(B, l^{\infty}\right):\|w\|_{B} \leq d\right\}, K_{\mathbb{R}^{n}}[r]=\left\{q \in \mathbb{R}^{n}:\|q\| \leq r\right\}$.

Assumption $H_{\star}[F]$. The function $f: \Omega \rightarrow \mathbb{S}$ satisfies Assumption $H_{0}[F]$ and there is $\gamma \in \mathbb{L}\left([0, a], \mathbb{R}_{+}\right)$such that the terms

$$
\begin{aligned}
& \left\|\partial_{x} F(t, x, v, w, q)-\partial_{x} F(t, \bar{x}, \bar{v}, \bar{w}, \bar{q})\right\|_{\infty \times n}, \quad \| \partial_{q} F(t, x, v, w, q) \\
& \quad-\partial_{q} F(t, \bar{x}, \bar{v}, \bar{w}, \bar{q}) \|_{\infty \times n}
\end{aligned}
$$

and

$$
\begin{aligned}
& \left\|\partial_{v} F(t, x, v, w, q)-\partial_{v} F(t, \bar{x}, \bar{v}, \bar{w}, \bar{q})\right\|_{\infty \times \infty ; \star}, \quad \| \partial_{w} F(t, x, v, w, q) \\
& \quad-\partial_{w} F(t, \bar{x}, \bar{v}, \bar{w}, \bar{q}) \|_{\infty \times \infty ; \star}
\end{aligned}
$$

are bounded from above by $\gamma(t)\left[\|x-\bar{x}\|+\|v-\bar{v}\|_{B}+\|w-\bar{w}\|_{B}+\|q-\bar{q}\|\right]$ on $\Omega[d, r]$.

Remark 2.2 It is important in our considerations that we have assumed the Lipschitz condition for $\partial_{x} F, \partial_{v} F, \partial_{w} F, \partial_{q} F$ with respect to $(x, v, w, q)$ and the estimates are local with respect to all variables. It is clear that there are differential systems with deviated variables and differential integral systems such that local estimates hold and global inequalities are not satisfied.

Lemma 2.3 Suppose that Assumptions $H_{\star}[F], H[\varphi]$ are satisfied and $\kappa<c \leq a$ and

$$
\begin{aligned}
& \psi, \bar{\psi} \in \mathbb{X}, \quad z \in C_{\psi \cdot c}\left[\zeta, d_{0}\right], \quad \bar{z} \in C_{\bar{\psi} . c}\left[\zeta, d_{0}\right], \\
& u, \bar{u} \in C\left(E_{c}, M_{\infty \times n}\right), \quad u=\left[u_{i j}\right]_{i \in \mathbb{N}, 1 \leq j \leq n}, \quad \bar{u}=\left[\bar{u}_{i j}\right]_{i \in \mathbb{N}, 1 \leq j \leq n}, \\
& u_{[i]}=\left(u_{i 1}, \ldots, u_{i n}\right), \quad \bar{u}_{[i]}=\left(\bar{u}_{i 1}, \ldots, \bar{u}_{i n}\right), \quad i \in \mathbb{N},
\end{aligned}
$$

and $u_{[i]} \in C_{\partial \psi_{i} . c}\left[\chi, r_{0}\right], \bar{u}_{[i]} \in C_{\partial \bar{\psi}_{i . c}}\left[\chi, r_{0}\right]$ for $i \in \mathbb{N}$. 
Then for each $k \in \mathbb{N}$ we have:

(i) the bicharacteristics $g_{[k]}\left[z, u_{[k]}\right](\cdot, t, x)$ and $g_{[k]}\left[\bar{z}, \bar{u}_{[k]}\right](\cdot, t, x),(t, x) \in E \cap\left(\left[a_{k}, c\right] \times\right.$ $\left.\mathbb{R}^{n}\right)$, exist on intervals $\left[a_{k}, \Delta\left[z, u_{[k]}\right](t, x)\right]$ and $\left[a_{k}, \Delta\left[\bar{z}, \bar{u}_{[k]}\right](t, x)\right]$ such that for $\tau=\Delta\left[z, u_{[k]}\right](t, x), \bar{\tau}=\Delta\left[\bar{z}, \bar{u}_{[k]}\right](t, x)$ we have $\left(\tau, g_{[k]}\left[z, u_{[k]}\right](\tau, t, x)\right) \in \partial E_{c}$, $\left(\bar{\tau}, g_{[k]}\left[\bar{z}, \bar{u}_{[k]}\right](\bar{\tau}, t, x)\right) \in \partial E_{c}$, where $\partial E_{c}$ is the boundary of $E_{c}$,

(ii) for each $k \in \mathbb{N}$ the solution of (9) is unique and we have the estimates

$$
\begin{aligned}
& \left\|g_{[k]}\left[z, u_{[k]}\right](\tau, t, x)-g_{[k]}\left[z, u_{[k]}\right](\tau, \bar{t}, \bar{x})\right\| \\
& \leq\left[\|x-\bar{x}\|+\left|\int_{t}^{\bar{t}}\|L(\xi)\| d \xi\right|\right] \exp \left\{\bar{C}\left|\int_{\tau}^{t} \gamma(\xi) d \xi\right|\right\},
\end{aligned}
$$

and

$$
\begin{aligned}
& \left\|g_{[k]}\left[z, u_{[k]}\right](\tau, t, x)-g_{[k]}\left[\bar{z}, \bar{u}_{[k]}\right](\tau, t, x)\right\| \\
& \quad \leq\left|\int_{\tau}^{t} \gamma(\xi)\left[2\|z-\bar{z}\|_{\left(\xi, l^{\infty}\right)}+\left\|u_{[k]}-\bar{u}_{[k]}\right\|_{\left(\xi, \mathbb{R}^{n}\right)}\right] d \xi\right| \exp \left\{\bar{C}\left|\int_{t}^{\tau} \gamma(\xi) d \xi\right|\right\},
\end{aligned}
$$

where $(t, x),(\bar{t}, \bar{x}) \in E \cap\left(\left[a_{k}, c\right] \times \mathbb{R}^{n}\right)$ and $\bar{C}=1+d_{0}\left(1+Q_{0}\right)+r_{0}$.

Proof The local existence and uniqueness of the solution to (9) follows from classical theorems on Carethéodory solutions of ordinary differential equations. Suppose that $\left[t_{0}, t\right]$ is the interval on which the bicharacteristic $g_{[k]}\left[z, u_{[k]}\right](\cdot, t, x)$ is defined. Then

$$
-L(\tau) \leq \frac{d}{d \tau} g_{[k]}\left[z, u_{[k]}\right](\tau, t, x) \leq L(\tau), \quad \tau \in\left[t_{0}, t\right],
$$

and consequently

$$
-b+M(\tau) \leq g_{[k]}\left[z, u_{[k]}\right](\tau, t, x) \leq b-M(\tau), \quad \tau \in\left[t_{0}, t\right] .
$$

We conclude that $\left(\tau, g_{[k]}\left[z, u_{[k]}\right](\tau, t, x)\right) \in E_{c}$ for $\tau \in\left[t_{0}, t\right]$ and the bicharacteristic $g_{[k]}\left[z, u_{[k]}\right](\cdot, t, x)$ is defined on $\left[a_{k}, t\right]$ and the assertion (i) follows.

Now we prove that for each $k \in \mathbb{N}$ the function $g_{[k]}\left[z, u_{[k]}\right](\cdot, t, x)-g_{[k]}\left[z, u_{[k]}\right](\cdot, \bar{t}, \bar{x})$ satisfies a linear integral inequality. Note that the functions $z_{(\tau, y)}$ and $z_{(\tau, \bar{y})}$ where $(\tau, y),(\tau, \bar{y}) \in E \cap\left([0, c] \times \mathbb{R}^{n}\right), y \neq \bar{y}$, have different domains. Hence we need the following construction. Write $B_{\star}=\left[-b_{0}, c\right] \times\left[-2 b+2 b^{-}, 2 b+2 b^{+}\right]$. There is $z_{\star} \in C\left(B_{\star}, l^{\infty}\right)$ such that

(i) $z_{\star}(t, x)=z(t, x)$ on $E_{c}$ and $\left\|\left(z_{\star}\right)_{(t, x)}\right\|_{B} \leq d$ on $E \cap\left([0, c] \times \mathbb{R}^{n}\right)$,

(ii) $\left\|z_{\star}(t, x)-z_{\star}(t, \bar{x})\right\|_{\infty} \leq d_{0}\|x-\bar{x}\|$ on $E \cap\left([0, c] \times \mathbb{R}^{n}\right)$.

Then the functions $\left(z_{\star}\right)_{(\tau, y)}$ and $\left(z_{\star}\right)_{(\tau, \bar{y})}$ where $(\tau, y),(\tau, \bar{y}) \in E \cap\left([0, c] \times \mathbb{R}^{n}\right)$ are defined on $B$. It follows form (9) that

$$
\begin{aligned}
& g_{[k]}\left[z, u_{[k]}\right](\tau, t, x)-g_{[k]}\left[z, u_{[k]}\right](\tau, \bar{t}, \bar{x})=x-\bar{x} \\
& \quad+\int_{\tau}^{t} \partial_{q} F_{k}\left(P\left[z_{\star}, u_{[k]}\right](\xi, t, x)\right) d \xi-\int_{\tau} \partial_{q} F_{k}\left(P\left[z_{\star}, u_{[k]}\right](\xi, \bar{t}, \bar{x})\right) d \xi
\end{aligned}
$$


and consequently

$$
\begin{aligned}
& \left\|g_{[k]}\left[z, u_{[k]}\right](\tau, t, x)-g_{[k]}\left[z, u_{[k]}\right](\tau, \bar{t}, \bar{x})\right\| \\
& \leq\|x-\bar{x}\|+\left|\int_{t}^{\bar{t}}\|L(\xi)\| d \xi\right|+\bar{C}\left|\int_{\tau}^{t} \gamma(\xi)\left\|g_{[k]}\left[z, u_{[k]}\right](\xi, t, x)-g_{[k]}\left[z, u_{[k]}\right](\xi, \bar{t}, \bar{x})\right\| d \xi\right| .
\end{aligned}
$$

From the Gronwall inequality we deduce (11). It follows from Assumptions $H[\varphi], H_{0}[F]$ and from (9) that

$$
\begin{aligned}
& \left\|g_{[k]}\left[z, u_{[k]}\right](\tau, t, x)-g_{[k]}\left[\bar{z}, \bar{u}_{[k]}\right](\tau, t, x)\right\| \\
& \leq\left|\int_{\tau}^{t} \gamma(\xi)\left[2\|z-\bar{z}\|_{\left(\xi, l^{\infty}\right)}+\left\|u_{[k]}-\bar{u}_{[k]}\right\|_{\left(\xi, \mathbb{R}^{n}\right)}\right] d \xi\right| \\
& \quad+\bar{C}\left|\int_{\tau}^{t} \gamma(\xi)\left\|g_{[k]}\left[z, u_{[k]}\right](\xi, t, x)-g_{[k]}\left[\bar{z}, \bar{u}_{[k]}\right](\xi, t, x)\right\| d \xi\right| .
\end{aligned}
$$

Then we obtain (12) form the Gronwall inequality.

Remark 2.4 Set $\bar{\Omega}=E \times C\left(B, l^{\infty}\right) \times \mathbb{R}^{n}$ and suppose that $\bar{F}: \bar{\Omega} \rightarrow \mathbb{S}, \bar{F}=\left\{\bar{F}_{k}\right\}_{k \in \mathbb{N}}$, is a given function of the variables $(t, x, v, q)$. Let us consider the functional differential system

$$
\partial_{t} z_{k}(t, x)=\bar{F}_{k}\left(t, x, z_{(t, x)}, \partial_{x} z_{k}(t, x)\right), \quad k \in \mathbb{N},
$$

which is a particular case of (1).

There are the following motivation for investigations of (1), (2) instead of (13), (2). Differential equations with deviated variables are obtained from (13) in the following way. Suppose that $G: E \times l^{\infty} \times l^{\infty} \times \mathbb{R}^{n} \rightarrow \mathbb{S}, G=\left\{G_{k}\right\}_{k \in \mathbb{N}}$, is a given function. Write

$$
\bar{F}(t, x, v, q)=G\left(t, x, v\left(0,0_{[n]}\right), v(\varphi(t, x)-(t, x)), q\right) .
$$

Then system (13) is equivalent to (4).

Note that Assumption $H_{0}[F]$ is not satisfied in this case for $\bar{F}$ given by (14). More precisely, the derivatives

$$
\partial_{x} \bar{F}(t, x, v, q)=\left[\partial_{x_{j}} \bar{F}_{i}(t, x, v, q)\right]_{i \in \mathbb{N}, 1 \leq j \leq n}
$$

do not exist on $\bar{\Omega}$. It is clear that under natural assumptions on $G$ the function $F$ given by (3) satisfies Assumption $H_{0}[F]$.

Let us consider the second example. Suppose that $E$ and $E_{0}$ are given by (5) and (6) respectively. For the above $G$ we put

$$
\bar{F}(t, x, v, q)=G\left(t, x, \int_{-h}^{h} v(\mu, y) d y, v(\varphi(t, x)-(t, x)), q\right) \text { on } \bar{\Omega} .
$$

Then system (13) is equivalent to (8). Note that Assumption $H_{0}[F]$ is not satisfied for $\bar{F}$ given by (16) because the derivatives (15) do not exist on $\bar{\Omega}$. It is clear that under natural assumptions on $G$ the function $F$ given by (7) satisfies Assumption $H_{0}[F]$.

With the above motivation we have considered problem (1), (2). 


\section{Existence of solutions to initial problems}

For $\psi \in \mathbb{X}, \psi=\left\{\psi_{k}\right\}_{k \in \mathbb{N}}$, we put

$$
\begin{aligned}
& \left\|\psi_{k}\right\|_{E_{0 . k}}=\max \left\{\left|\psi_{k}(t, x)\right|:(t, x) \in E_{0 . k}\right\}, \\
& \left\|\partial_{x} \psi_{k}\right\|_{E_{0 . k}}=\max \left\{\left\|\partial_{x} \psi_{k}(t, x)\right\|:(t, x) \in E_{0 . k}\right\}
\end{aligned}
$$

where $k \in \mathbb{N}$ and

$$
\|\psi\|_{\mathbb{X}}=\sup \left\{\left\|\psi_{k}\right\|_{E_{0 . k}}+\left\|\partial_{x} \psi_{k}\right\|_{E_{0 . k}}: k \in \mathbb{N}\right\}
$$

Write

$$
\begin{aligned}
& \hat{\Gamma}(t)=c_{1}+\left(1+d_{0}+d_{0} Q_{0}\right) \int_{0}^{t} \beta(\xi) d \xi+r \bar{C} \int_{0}^{t} \gamma(\xi) d \xi+2 r_{0} \int_{0}^{t}\|L(\xi)\| d \xi, \\
& \Gamma(t)=\hat{\Gamma}(t) \exp \left[\bar{C} \int_{0}^{t} \gamma(\xi) d \xi\right], \\
& \left.\Lambda(t)=\left\{\begin{array}{c}
c_{2}+B_{0} \int_{0}^{t} \gamma(\xi) d \xi+B_{1} \int_{0}^{t} \beta(\xi) d \xi \\
B_{0}=\bar{C}\left(1+r+r Q_{0}\right), \quad B_{1}=r_{0}+r_{0} Q_{0}^{2}+r Q .
\end{array}\right] \bar{C} \int_{0}^{t} \gamma(\xi) d \xi\right],
\end{aligned}
$$

Assumption $H[F]$. The function $F: \Omega \rightarrow \mathbb{S}$ satisfies Assumption $H_{\star}[F]$ and the constant $c \in(\kappa, a]$ is small enough to satisfy the conditions

$$
\Gamma(c) \leq d_{0}, \quad \Lambda(c) \leq r_{0} .
$$

Remark 3.1 If we assume that $d_{0}>c_{1}$ and $r_{0}>c_{2}$ then there is $c \in(0, a]$ such that condition (17) is satisfied.

Theorem 3.2 IfAssumption $H[\varphi], H[F]$ are satisfied and $\psi \in \mathbb{X}$ then there exists a solution $\hat{z}: E_{c} \rightarrow l^{\infty}$ to (1), (2) and

$$
\|\hat{z}\|_{\left(t, l^{\infty}\right)} \leq d, \quad\left\|\partial_{x} \hat{z}\right\|_{\left(t, M_{\infty \times n}\right)} \leq r \quad \text { for } t \in[0, c],
$$

and

$$
\left\|\partial_{x} \hat{z}(t, x)-\partial_{x} \hat{z}(t, \bar{x})\right\|_{\infty \times n} \leq r_{0}\|x-\bar{x}\| \quad \text { on } E_{c} .
$$

If $\tilde{\psi} \in \mathbb{X}, \tilde{\psi}=\left\{\tilde{\psi}_{k}\right\}_{k \in \mathbb{N}}$, and $\tilde{z}: E_{c} \rightarrow l^{\infty}$ is a solution to (1) with the initial conditions

$$
z_{k}(t, x)=\tilde{\psi}_{k}(t, x) \text { on } E_{0 . k} \quad \text { for } k \in \mathbb{N} \text {, }
$$

then

$$
\|\hat{z}-\tilde{z}\|_{\left(t, l^{\infty}\right)}+\left\|\partial_{x} \hat{z}-\partial_{x} \tilde{z}\right\|_{\left(t, M_{\infty \times n}\right)} \leq\|\psi-\tilde{\psi}\|_{\mathbb{X}} \exp \left[\int_{0}^{t} \Gamma_{\star}(\tau) d \tau\right], \quad t \in[0, c],
$$

where

$$
\Gamma_{\star}(\tau)=B_{\star} \gamma(\tau)+2 \beta(\tau)+2\|L(\tau)\|, \quad B_{\star}=2\left[\Gamma(c)+\Lambda(c)+1+r+r Q_{0}\right] .
$$


Proof We have divided the proof into a sequence of steps. We use a method of successive approximations.

I. We consider the sequences $\left\{z^{(m)}\right\}$ and $\left\{u^{(m)}\right\}$ where

$$
\begin{aligned}
& z^{(m)}: E_{c} \rightarrow l^{\infty}, \quad u^{(m)}: E_{c} \rightarrow M_{\infty \times n}, \\
& z^{(m)}=\left\{z_{k}^{(m)}\right\}_{k \in \mathbb{N}}, \quad u^{(m)}=\left\{u_{[k]}^{(m)}\right\}_{k \in \mathbb{N}}, \quad u_{[k]}^{(m)}=\left(u_{k 1}^{(m)}, \ldots, u_{k n}^{(m)}\right), \quad k \in \mathbb{N} .
\end{aligned}
$$

We put first

$$
z_{k}^{(0)}(t, x)=\psi_{k}(t, x) \text { on } E_{0 . k} \text { and } z_{k}^{(0)}=\psi_{k}\left(a_{k}, x\right) \text { on } E \cap\left(\left[a_{k}, c\right] \times \mathbb{R}^{n}\right) \text { for } k \in \mathbb{N},
$$

and

$$
u_{k}^{(0)}(t, x)=\partial_{x} \psi_{k}\left(a_{k}, x\right) \text { on } E \cap\left(\left[a_{k}, c\right] \times \mathbb{R}^{n}\right) \text { for } k \in \mathbb{N} .
$$

If $z^{(m)}: E_{c} \rightarrow l^{\infty}$ and $u^{(m)}: E_{c} \rightarrow M_{\infty \times n}$ are known functions then for each $k \in \mathbb{N}$ the function $u_{[k]}^{(m+1)}$ is a solution of the equation

$$
\vartheta(t, x)=\mathbb{G}_{k}^{(m)}[\vartheta](t, x)
$$

where $\vartheta=\left(\vartheta_{1}, \ldots, \vartheta_{n}\right)$ and $\mathbb{G}_{k}^{(m)}[\vartheta]=\left(\mathbb{G}_{k 1}^{(m)}[\vartheta], \ldots, \mathbb{G}_{k n}^{(m)}[\vartheta]\right)$ and $\mathbb{G}_{k}^{(m)}[\vartheta]$ is defined by

$$
\mathbb{G}_{k}^{(m)}[\vartheta](t, x)=\partial_{x} \psi_{k}(t, x) \text { on } E_{0 . k}
$$

and

$$
\begin{aligned}
& \mathbb{G}_{k}^{(m)}[\vartheta](t, x)=\partial_{x} \psi_{k}\left(a_{k}, g_{[k]}\left[z^{(m)}, \vartheta\right]\left(a_{k}, t, x\right)\right) \\
& \quad+\int_{a_{k}}^{t} \partial_{x} F_{k}\left(P\left[z^{(m)}, \vartheta\right](\tau, t, x)\right) d \tau+\int_{a_{k}}^{t} \partial_{v} F_{k}\left(P\left[z^{(m)}, \vartheta\right](\tau, t, x)\right) \star u_{\left(\tau, g_{[k]}\left[z^{(m)}, \vartheta\right](\tau, t, x)\right)}^{(m)} d \tau \\
& \quad+\int_{a_{k}}^{t} \partial_{w} F_{k}\left(P\left[z^{(m)}, \vartheta\right](\tau, t, x)\right) \star\left[u_{\varphi\left(\tau, g_{[k]}\left[z^{(m)}, \vartheta\right](\tau, t, x)\right)}^{(m)} \partial_{x} \phi\left(\tau, g_{[k]}\left[z^{(m)}, \vartheta\right](\tau, t, x)\right)\right] d \tau
\end{aligned}
$$

on $E \cap\left(\left[a_{k}, c\right] \times \mathbb{R}^{n}\right)$. The function $z^{(m+1)}$ is given by

$$
z^{(m+1)}(t, x)=\mathbb{F}\left[z^{(m)}, u^{(m+1)}\right](t, x) \quad \text { on } E_{c} .
$$

II. We prove that

$\left(A_{m}\right)$ the sequences $\left\{z^{(m)}\right\}$ and $\left\{u^{(m)}\right\}$ are defined on $E_{c}$ and for $m \geq 0$ we have

$$
z^{(m)} \in C_{\psi . c}\left[\zeta, d_{0}\right], \quad u_{k}^{(m)} \in C_{\partial \psi_{k} . c}\left[\chi, r_{0}\right] \text { for } k \in \mathbb{N},
$$

$\left(B_{m}\right)$ there are $\lambda, \lambda_{0} \in \mathbb{L}\left([0, c], \mathbb{R}_{+}\right)$such that for any $m \geq 0$ we have

$\left\|z^{(m)}(t, x)-z^{(m)}(\tilde{t}, x)\right\|_{\infty} \leq\left|\int_{t}^{\tilde{t}} \lambda_{0}(\tau) d \tau\right|, \quad\left\|u^{(m)}(t, x)-u^{(m)}(\tilde{t}, x)\right\|_{\infty \times n} \leq\left|\int_{t}^{\tilde{t}} \lambda(\tau) d \tau\right|$,

where $(t, x),(\tilde{t}, x) \in E_{c}, 0 \leq t, \tilde{t} \leq c$,

$\left(C_{m}\right)$ there exists the sequence $\left\{\partial_{x} z^{(m)}\right\}$ and for $m \geq 0$ we have: $\partial_{x} z^{(m)}(t, x)=u^{(m)}(t, x)$ on $E_{c}$. 
We prove $\left(A_{m}\right)-\left(C_{m}\right)$ by induction. It is clear that conditions $\left(A_{0}\right)-\left(C_{0}\right)$ are satisfied. Supposed now that $\left(A_{m}\right)-\left(C_{m}\right)$ hold for a given $m \geq 0$, we will prove that there exists $u^{(m+1)}: E_{c} \rightarrow M_{\infty \times n}$ and $u_{[k]}^{(m+1)} \in C_{\partial \psi_{k} . c}\left[\chi, r_{0}\right]$ for $k \in \mathbb{N}$. We first prove that

$$
\mathbb{G}_{k}^{(m)}: C_{\partial \psi_{k} . c}\left[\chi, r_{0}\right] \rightarrow C_{\partial \psi_{k} . c}\left[\chi, r_{0}\right], \quad k \in \mathbb{N} .
$$

It follows from Assumptions $H[\varphi]$ and $H[F]$ that for $\vartheta \in C_{\partial \psi_{k} . c}\left[\chi, r_{0}\right], k \in \mathbb{N}$, we have

$$
\left\|\mathbb{G}_{k}^{(m)}[\vartheta](t, x)\right\| \leq c_{1}+\left(1+Q_{0}\right) \int_{0}^{t} \beta(\xi) \chi(\xi) d \xi+\int_{0}^{t} \beta(\xi) d \xi=\chi(t)
$$

and

$$
\left\|\mathbb{G}_{k}^{(m)}[\vartheta](t, x)-\mathbb{G}_{k}^{(m)}[\vartheta](t, \tilde{x})\right\| \leq \Lambda(t)\|x-\tilde{x}\| \leq r_{0}\|x-\tilde{x}\| \quad \text { on } E_{c} \cap\left(\left[a_{k}, c\right] \times \mathbb{R}^{n}\right) .
$$

From the above estimates we deduce (25). It follows easily that for $\vartheta, \tilde{\vartheta} \in C_{\partial \psi_{k} . c}\left[\chi, r_{0}\right]$ we have

$$
\left\|\mathbb{G}_{k}^{(m)}[\vartheta](t, x)-\mathbb{G}_{k}^{(m)}[\tilde{\vartheta}](t, x)\right\| \leq \Lambda(c) \int_{a_{k}}^{t} \gamma(\xi)\|\vartheta-\tilde{\vartheta}\|_{\left(\xi, \mathbb{R}^{n}\right)} d \xi \quad \text { on } E \cap\left(\left[a_{k}, c\right] \times \mathbb{R}^{n}\right) .
$$

For the above $\vartheta, \tilde{\vartheta}$ we put

$$
[|\vartheta-\tilde{\vartheta}|]=\max \left\{\|\vartheta-\tilde{\vartheta}\|_{\left(t, \mathbb{R}^{n}\right)} \exp \left[-2 \Lambda(c) \int_{a_{k}}^{t} \gamma(\xi) d \xi\right]: t \in\left[a_{k}, c\right]\right\} .
$$

We deduce from Assumption $H[F]$ that

$$
\left[\left|\mathbb{G}_{k}^{(m)}[\vartheta]-\mathbb{G}_{k}^{(m)}[\tilde{\vartheta}]\right|\right] \leq \frac{1}{2}[|\vartheta-\tilde{\vartheta}|] .
$$

It follows from the Banach fixed point theorem that for each $k \in \mathbb{N}$ there exists exactly one solution of Eq. (22). Then there exists $u^{(m+1)}: E_{c} \rightarrow M_{\infty \times n}$ and $u_{k}^{(m+1)} \in C_{\partial \psi_{k} . c}\left[\chi, r_{0}\right]$ for $k \in \mathbb{N}$.

We deduce from Assumption $H[\varphi], H[F]$ and from (24) that

$$
\left\|z^{(m+1)}\right\|_{(t, \infty)} \leq c_{0}+\int_{0}^{t} \alpha(\tau) d \tau+2 \int_{0}^{t} \beta(\tau) \zeta(\tau) d \tau+2 \int_{0}^{t}\|L(\tau)\| \chi(\tau) d \tau=\zeta(t)
$$

where $t \in[0, c]$ and

$$
\left|z_{k}^{(m+1)}(t, x)-z_{k}^{(m+1)}(t, \tilde{x})\right| \leq \Gamma(t)\|x-\tilde{x}\| \quad \text { on } E \cap\left(\left[a_{k}, c\right] \times \mathbb{R}^{n}\right) .
$$

The above relations and Assumption $H[c]$ show that $z^{(m+1)} \in C_{\psi . c}\left[\zeta, d_{0}\right]$.

An easy computation shows that condition $\left(B_{m+1}\right)$ is satisfied with

$$
\begin{aligned}
& \lambda_{0}(\tau)=(\Gamma(c)+2 r)\|L(\tau)\|+2 d \beta(\tau)+\alpha(\tau), \\
& \lambda(\tau)=\Lambda(c)\|L(\tau)\|+\left(1+r+r Q_{0}\right) \beta(\tau), \quad \tau \in[0, c] .
\end{aligned}
$$

Now we prove $\left(C_{m+1}\right)$. Write

$$
\mathbb{D}_{k}(t, x, y)=z_{k}^{(m+1)}(t, y)-z_{k}^{(m+1)}(t, x)-u_{[k]}^{(m+1)}(t, x) \circ(y-x), \quad k \in \mathbb{N},
$$


where $(t, x),(t, y) \in E \cap\left(\left[a_{k}, c\right] \times \mathbb{R}^{n}\right)$. We prove that there is $C_{\star} \in \mathbb{R}_{+}$such that

$$
\left|\mathbb{D}_{k}(t, x, y)\right| \leq C_{\star}\|x-y\|^{2}, \quad k \in \mathbb{N} .
$$

Set $g_{[k]}^{(m)}(\tau, t, x)=g_{[k]}\left[z^{(m)}, u_{[k]}^{(m+1)}\right](\tau, t, x)$. Then we have

$$
\begin{aligned}
\mathbb{D}_{k}(t, x, y)= & \mathbb{F}_{k}\left[z^{(m)}, u^{(m+1)}\right](t, y)-\mathbb{F}\left[z^{(m)}, u^{(m+1)}\right](t, x)-\mathbb{G}_{[k]}\left[u_{[k]}^{(m+1)}\right](t, x) \circ(y-x) \\
= & \psi_{k}\left(a_{k}, g_{[k]}^{(m)}\left(a_{k}, t, y\right)\right)-\psi_{k}\left(a_{k}, g_{[k]}^{(m)}\left(a_{k}, t, x\right)\right) \\
& +\int_{a_{k}}^{t}\left[F_{k}\left(P\left[z^{(m)}, u_{[k]}^{(m+1)}\right](\tau, t, y)\right)-F_{k}\left(P\left[z^{(m)}, u_{[k]}^{(m+1)}\right](\tau, t, x)\right)\right] d \tau \\
& -\int_{a_{k}}^{t} \partial_{q} F_{k}\left(P\left[z^{(m)}, u_{[k]}^{(m+1)}\right](\tau, t, y)\right) \circ u_{[k]}^{(m+1)}\left(\tau, g_{[k]}^{(m)}(\tau, t, y)\right) d \tau \\
& +\int_{a_{k}}^{t} \partial_{q} F_{k}\left(P\left[z^{(m)}, u_{[k]}^{(m+1)}\right](\tau, t, x)\right) \circ u_{[k]}^{(m+1)}\left(\tau, g_{[k]}^{(m)}(\tau, t, x)\right) d \tau \\
& -\mathbb{G}_{[k]}\left[u_{[k]}^{(m+1)}\right](t, x) \circ(y-x), \quad k \in \mathbb{N} .
\end{aligned}
$$

We transform the above expressions in the following way. We apply the Hadamard mean value theorem to the differences

$$
F_{k}\left(P\left[z^{(m)}, u_{[k]}^{(m+1)}\right](\tau, t, y)\right)-F_{k}\left(P\left[z^{(m)}, u_{[k]}^{(m+1)}\right](\tau, t, x)\right), \quad k \in \mathbb{N},
$$

and we denote by

$$
\begin{aligned}
& Q^{(m)}(\xi, \tau, t, x, y)=\xi P\left[z^{(m)}, u_{[k]}^{(m+1)}\right](\tau, t, y) \\
& \quad+(1-\xi) P\left[z^{(m)}, u_{[k]}^{(m+1)}\right](\tau, t, x), \quad \xi \in[0,1], k \in \mathbb{N},
\end{aligned}
$$

suitable intermediate points. Let us denote by $\mathbb{D}_{k .0}(t, x, y), \mathbb{D}_{k .1}(t, x, y), \mathbb{D}_{k .2}(t, x, y)$, $\mathbb{D}_{k .3}(t, x, y)$ the expressions defined by

$$
\begin{aligned}
& \mathbb{D}_{k .0}(t, x, y)=\psi_{k}\left(a_{k}, g_{[k]}^{(m)}\left(a_{k}, t, y\right)\right)-\psi_{k}\left(a_{k}, g_{[k]}^{(m)}\left(a_{k}, t, x\right)\right) \\
& \quad-\partial_{x} \psi_{k}\left(a_{k}, g_{[k]}^{(m)}\left(a_{k}, t, x\right)\right) \circ\left[g_{[k]}^{(m)}\left(a_{k}, t, y\right)-g_{[k]}^{(m)}\left(a_{k}, t, x\right)\right], \\
& \mathbb{D}_{k .1}(t, x, y)=\int_{a_{k}}^{t} \int_{0}^{1}\left[\partial_{x} F_{k}\left(Q^{(m)}(\xi, \tau, t, x, y)\right)\right. \\
& \left.\quad-\partial_{x} F_{k}\left(P\left[z^{(m)}, u_{[k]}^{(m+1)}\right](\tau, t, x)\right)\right] \circ\left[g_{[k]}^{(m)}(\tau, t, y)-g_{[k]}^{(m)}(\tau, t, x)\right] d \xi d \tau \\
& +\int_{a_{k}}^{t} \int_{0}^{1}\left[\partial_{v} F_{k}\left(Q^{(m)}(\xi, \tau, t, x, y)\right)-\partial_{v} F_{k}\left(P\left[z^{(m)}, u_{[k]}^{(m+1)}\right](\tau, t, x)\right)\right] \\
& \star \\
& \quad\left[z_{\left(\tau, g_{[k]}^{(m)}(\tau, t, y)\right)}^{(m)}-z_{\left(\tau, g_{[k]}^{(m)}(\tau, t, x)\right)}^{(m)}\right] d \xi d \tau \\
& +\int_{a_{k}}^{t} \int_{0}^{1}\left[\partial_{w} F_{k}\left(Q^{(m)}(\xi, \tau, t, x, y)\right)-\partial_{w} F_{k}\left(P\left[z^{(m)}, u_{[k]}^{(m+1)}\right](\tau, t, x)\right)\right]
\end{aligned}
$$




$$
\begin{aligned}
& \star\left[z_{\varphi\left(\tau, g_{[k]}^{(m)}(\tau, t, y)\right)}^{(m)}-z_{\varphi\left(\tau, g_{[k]}^{(m)}(\tau, t, x)\right)}^{(m)}\right] d \xi d \tau \\
& +\int_{a_{k}}^{t} \int_{0}^{1}\left[\partial_{q} F_{k}\left(Q^{(m)}(\xi, \tau, t, x, y)\right)-\partial_{q} F_{k}\left(P\left[z^{(m)}, u_{[k]}^{(m+1)}\right](\tau, t, x)\right)\right] \\
& \circ\left[u_{[k]}^{(m+1)}\left(\tau, g_{[k]}^{(m)}(\tau, t, y)\right)-u_{[k]}^{(m+1)}\left(\tau, g_{[k]}^{(m)}(\tau, t, x)\right)\right] d \xi d \tau
\end{aligned}
$$

and

$$
\begin{aligned}
& \mathbb{D}_{k .2}(t, x, y)=\int_{a_{k}}^{t} \partial_{v} F_{k}\left(P\left[z^{(m)}, u_{[k]}^{(m+1)}\right](\tau, t, x)\right) \star\left[z_{\left(\tau, g_{[k]}^{(m)}(\tau, t, y)\right)}^{(m)}-\left(u^{(m)}\right)_{\left(\tau, g_{[k]}^{(m)}(\tau, t, x)\right)}\left(g_{[k]}^{(m)}(\tau, t, y)-g_{[k]}^{(m)}(\tau, t, x)\right)^{T}\right] d \tau \\
& -z_{\left(\tau, g_{[k]}^{(m)}(\tau, t, x)\right)}^{(m)}-{ }^{t} \int_{a_{k}} \partial_{w} F_{k}\left(P\left[z^{(m)}, u_{[k]}^{(m+1)}\right](\tau, t, x)\right) \star\left[z_{\varphi\left(\tau, g_{[k]}^{(m)}(\tau, t, y)\right)}^{(m)}-z_{\varphi\left(\tau, g_{[k]}^{(m)}(\tau, t, x)\right)}^{(m)}\right. \\
& \left.-\left(u^{(m)}\right)_{\varphi\left(\tau, g_{[k]}^{(m)}(\tau, t, x)\right)} \partial_{x} \phi\left(\tau, g_{[k]}^{(m)}(\tau, t, x)\right)\left(g_{[k]}^{(m)}(\tau, t, y)-g_{[k]}^{(m)}(\tau, t, x)\right)^{T}\right] d \tau .
\end{aligned}
$$

\section{Moreover we set}

$$
\begin{aligned}
& \mathbb{D}_{k .3}(t, x, y)=\partial_{x} \psi_{k}\left(a_{k}, g_{[k]}^{(m)}\left(a_{k}, t, x\right)\right) \circ\left[\left[g_{[k]}^{(m)}\left(a_{k}, t, y\right)-g_{[k]}^{(m)}\left(a_{k}, t, x\right)\right]-(y-x)\right] \\
& \quad+\int_{a_{k}}^{t}\left[\partial_{q} F_{k}\left(P\left[z^{(m)}, u_{[k]}^{(m+1)}\right](\tau, t, x)\right)-\partial_{q} F_{k}\left(P\left[z^{(m)}, u_{[k]}^{(m+1)}\right](\tau, t, y)\right)\right] \circ u_{[k]}^{(m+1)} \\
& \quad \times\left(\tau, g_{[k]}^{(m)}(\tau, t, y)\right) d \tau \\
& \quad+\int_{a_{k}}^{t} \partial_{x} F_{k}\left(P\left[z^{(m)}, u_{[k]}^{(m+1)}\right](\tau, t, x)\right) \circ\left[\left[g_{[k]}^{(m)}(\tau, t, y)-g_{[k]}^{(m)}(\tau, t, x)\right]-(y-x)\right] d \tau \\
& \left.\quad+\int_{a_{k}}^{t} \partial_{v} F_{k}\left(P\left[z^{(m)}, u_{[k]}^{(m+1)}\right](\tau, t, x)\right) \star\left(u^{(m)}\right)_{\left(\tau, g_{[k]}^{(m)}(\tau, t, x)\right)}\right] \circ\left[\left[g_{[k]}^{(m)}(\tau, t, y)\right.\right. \\
& \left.\left.\quad-g_{[k]}^{(m)}(\tau, t, x)\right]-(y-x)\right] d \tau \\
& \quad+\int_{a_{k}}^{t}\left\{\partial_{w} F_{k}\left(P\left[z^{(m)}, u_{[k]}^{(m+1)}\right](\tau, t, x)\right) \star\left[\left(u^{(m)}\right)_{\varphi\left(\tau, g_{[k]}^{(m)}(\tau, t, x)\right)} \partial_{x} \phi\left(\tau, g_{[k]}^{(m)}(\tau, t, x)\right)\right]\right\} \\
& \quad \circ\left[\left[g_{[k]}^{(m)}(\tau, t, y)-g_{[k]}^{(m)}(\tau, t, x)\right]-(y-x)\right] d \tau .
\end{aligned}
$$

We put $k \in \mathbb{N}$ in the above definitions. Then we have

$$
\mathbb{D}_{k}(t, x, y)=\mathbb{D}_{k .0}(t, x, y)+\mathbb{D}_{k .1}(t, x, y)+\mathbb{D}_{k .2}(t, x, y)+\mathbb{D}_{k .3}(t, x, y), \quad k \in \mathbb{N} . \text { (27) }
$$

Since $\psi \in \mathbb{X}$, there is $C_{0} \in \mathbb{R}_{+}$such that

$$
\left|\mathbb{D}_{k .0}(t, x, y)\right| \leq C_{0}\|x-y\|^{2}, \quad k \in \mathbb{N},
$$


where $(t, x),(t, y) \in E \cap\left(\left[a_{k}, c\right] \times \mathbb{R}^{n}\right)$. It is easily seen that

$$
\left|\mathbb{D}_{k .1}(t, x, y)\right| \leq C_{1}\|x-y\|^{2}, \quad k \in \mathbb{N},
$$

where

$$
C_{1}=(\tilde{c} \bar{C})^{2} \int_{0}^{c} \gamma(\xi) d \xi, \quad \tilde{c}=\exp \left[\bar{C} \int_{0}^{c} \gamma(\xi) d \xi\right] .
$$

It follows from $\left(C_{m}\right)$ that there is $c_{\star} \in \mathbb{R}_{+}$such that

$$
\left|z_{k}^{(m)}(t, y)-z_{k}^{(m)}(t, x)-u_{[k]}^{(m)}(t, x) \circ(y-x)\right| \leq c_{\star}\|x-y\|^{2},
$$

where $(t, x),(t, y) \in E \cap\left(\left[a_{k}, c\right] \times \mathbb{R}^{n}\right)$. We conclude from Assumptions $H[F], H[\varphi]$ and from (11), (30) that there is $C_{2} \in \mathbb{R}_{+}$such that

$$
\left|\mathbb{D}_{k .2}(t, x, y)\right| \leq C_{2}\|x-y\|^{2}, k \in \mathbb{N},
$$

where $(t, x),(t, y) \in E \cap\left(\left[a_{k}, c\right] \times \mathbb{R}^{n}\right)$.

We transform the expressions $\mathbb{D}_{k .3}(t, x, y), k \in \mathbb{N}$, in the following way. Write

$$
\begin{aligned}
& \mathbb{V}_{k}(\xi, \tau, t, x, y)=\left\{\partial_{x} F_{k}\left(P\left[z^{(m)}, u_{[k]}^{(m+1)}\right](\tau, t, x)\right)\right. \\
& \quad+\partial_{v} F_{k}\left(P\left[z^{(m)}, u_{[k]}^{(m+1)}\right](\tau, t, x)\right) \star\left(u^{(m)}\right)_{\left(\tau, g_{[k]}^{(m)}(\tau, t, x)\right)} \\
& \left.\quad+\partial_{w} F_{k}\left(P\left[z^{(m)}, u_{[k]}^{(m+1)}\right](\tau, t, x)\right) \star\left[\left(u^{(m)}\right)_{\left(\tau, g_{[k]}^{(m)}(\tau, t, x)\right)} \partial_{x} \phi\left(\tau, g_{[k]}^{(m)}(\tau, t, x)\right)\right]\right\} \\
& \quad \circ\left[\partial_{q} F_{k}\left(P\left[z^{(m)}, u_{[k]}^{(m+1)}\right](\xi, t, y)\right)-\partial_{q} F_{k}\left(P\left[z^{(m)}, u_{[k]}^{(m+1)}\right](\xi, t, x)\right)\right]
\end{aligned}
$$

and

$$
\begin{aligned}
& \mathbb{W}_{k}(\xi, t, x)=\partial_{x} \psi_{k}\left(a_{k}, g_{[k]}^{(m)}\left(a_{k}, t, x\right)\right) \\
& \quad+\int_{a_{k}}^{\xi}\left[\partial_{x} F_{k}\left(P\left[z^{(m)}, u_{[k]}^{(m+1)}\right](\tau, t, x)\right)+\partial_{v} F_{k}\left(P\left[z^{(m)}, u_{[k]}^{(m+1)}\right](\tau, t, x)\right)\right. \\
& \left.\star\left(u^{(m)}\right)_{\left(\tau, g_{[k]}^{(m)}(\tau, t, x)\right)}\right] d \tau \\
& \quad+\int_{a_{k}}^{\xi} \partial_{w} F_{k}\left(P\left[z^{(m)}, u_{[k]}^{(m+1)}\right](\tau, t, x)\right) \star\left[\left(u^{(m)}\right)_{\left(\tau, g_{[k]}^{(m)}(\tau, t, x)\right)} \partial_{x} \phi\left(\tau, g_{[k]}^{(m)}(\tau, t, x)\right)\right] d \tau, \quad k \in \mathbb{N},
\end{aligned}
$$

and

$$
\begin{aligned}
& \mathbb{D}_{k .4}(t, x, y)=\int_{a_{k}}^{t} \int_{\tau}^{t} \mathbb{V}_{k}(\xi, \tau, t, x, y) d \xi d \tau \\
& +\partial_{x} \psi_{k}\left(a_{k}, g_{[k]}^{(m)}\left(a_{k}, t, x\right)\right) \circ \int_{a_{k}}^{t}\left[\partial_{q} F_{k}\left(P\left[z^{(m)}, u_{[k]}^{(m+1)}\right](\xi, t, y)\right)\right. \\
& \left.-\partial_{q} F_{k}\left(P\left[z^{(m)}, u_{[k]}^{(m+1)}\right](\xi, t, x)\right)\right] d \xi
\end{aligned}
$$


where $k \in \mathbb{N}$. Then we have

$$
\int_{a_{k}}^{t} \int_{\tau}^{t} \mathbb{V}_{k}(\xi, \tau, t, x, y) d \xi d \tau=\int_{a_{k}}^{t} \int_{a_{k}}^{\xi} \mathbb{V}_{k}(\xi, \tau, t, x, y) d \tau d \xi
$$

and consequently

$$
\begin{aligned}
& \mathbb{D}_{k .4}(t, x, y) \\
& =\int_{a_{k}}^{t} \mathbb{W}_{k}(\xi, t, x) \circ\left[\partial_{q} F_{k}\left(P\left[z^{(m)}, u_{[k]}^{(m+1)}\right](\xi, t, y)\right)-\partial_{q} F_{k}\left(P\left[z^{(m)}, u_{[k]}^{(m+1)}\right](\xi, t, x)\right)\right] d \xi .
\end{aligned}
$$

It is clear that the bicharacteristics satisfy the relations

$$
g_{[k]}^{(m)}\left(\tau, \xi, g_{[k]}^{(m)}(\xi, t, x)\right)=g_{[k]}^{(m)}(\tau, t, x), \quad k \in \mathbb{N},
$$

where $(t, x) \in E \cap\left(\left[a_{k}, c\right] \times \mathbb{R}^{n}\right)$. This gives

$$
u_{[k]}^{(m+1)}\left(\xi, g_{[k]}^{(m)}(\xi, t, x)\right)=\mathbb{W}_{k}(\xi, t, x), \quad k \in \mathbb{N} .
$$

We conclude from (32), (33) that

$$
\begin{aligned}
& \mathbb{D}_{k .3}(t, x, y)=\int_{a_{k}}^{t}\left[\partial_{q} F_{k}\left(P\left[z^{(m)}, u_{[k]}^{(m+1)}\right](\tau, t, x)\right)\right. \\
& \left.-\partial_{q} F_{k}\left(P\left[z^{(m)}, u_{[k]}^{(m+1)}\right](\tau, t, y)\right)\right] \circ\left[u_{[k]}^{(m+1)}\left(\tau, g_{[k]}^{(m)}(\tau, t, y)\right)\right. \\
& \left.-u_{[k]}^{(m+1)}\left(\tau, g_{[k]}^{(m)}(\tau, t, x)\right)\right] d \tau, \quad k \in \mathbb{N} .
\end{aligned}
$$

Hence, there is $C_{3} \in \mathbb{R}_{+}$such that

$$
\left|\mathbb{D}_{k .3}(t, x, y)\right| \leq C_{3}\|x-y\|^{2}, \quad k \in \mathbb{N},
$$

where $(t, x),(t, y) \in E \cap\left(\left[a_{k}, c\right] \times \mathbb{R}^{n}\right)$.

It follows from (27)-(29), (31), (34) that estimates (26) are satisfied with $C^{\star}=C_{0}+C_{1}+$ $C_{2}+C_{3}$. Hence, for each $k \in \mathbb{N}$ there exists $\partial_{x} z^{(m+1)}(t, x)$ for $(t, x) \in E \cap\left(\left[a_{k}, c\right] \times \mathbb{R}^{n}\right)$ and $\partial_{x} z^{(m+1)}=u_{[k]}^{(m+1)}$. This proves $\left(C_{m+1}\right)$. Thus $\left(A_{m}\right)-\left(C_{m}\right)$ follow by induction.

III. Now we prove that the sequences $\left\{z^{(m)}\right\}$ and $\left\{u^{(m)}\right\}$ are uniformly convergent on $E_{c}$. It follows from Assumptions $H[F], H[\varphi]$ and from (23) that there are $\Upsilon_{0}, \Upsilon_{1} \in \mathbb{L}\left([0, c], \mathbb{R}_{+}\right)$ such that

$$
\begin{aligned}
& \left\|u_{[k]}^{(m+1)}-u_{[k]}^{(m)}\right\|_{\left(t, \mathbb{R}^{n}\right)} \leq \int_{0}^{t} \Upsilon_{0}(\tau)\left\|u_{[k]}^{(m+1)}-u_{[k]}^{(m)}\right\|_{\left(\tau, \mathbb{R}^{n}\right)} d \tau \\
& \int_{0}^{t} \Upsilon_{1}(\tau)\left[\left\|z^{(m)}-z^{(m-1)}\right\|_{\left(\tau, \mathbb{R}^{n}\right)}+\left\|u^{(m)}-u^{(m-1)}\right\|_{\left(\tau, M_{\infty \times n}\right)}\right] d \tau, \quad k \in \mathbb{N} .
\end{aligned}
$$


By using the Gronwall inequality we get

$$
\begin{aligned}
& \left\|u_{[k]}^{(m+1)}-u_{[k]}^{(m)}\right\|_{\left(t, \mathbb{R}^{n}\right)} \\
& \leq \exp \left[\int_{0}^{t} \Upsilon_{0}(\tau) d \tau\right] \int_{0}^{t} \Upsilon_{1}(\tau)\left[\left\|z^{(m)}-z^{(m-1)}\right\|_{\left(\tau, \mathbb{R}^{n}\right)}+\left\|u^{(m)}-u^{(m-1)}\right\|_{\left(\tau, M_{\infty \times n}\right)}\right] d \tau,
\end{aligned}
$$

where $k \in \mathbb{N}$. We conclude from (10) and from Assumption $H[F], H[\varphi]$ that there is $\Upsilon_{2} \in \mathbb{L}\left([0, c], \mathbb{R}_{+}\right)$such that

$$
\begin{aligned}
& \left\|z^{(m+1)}-z^{(m)}\right\|_{\left(t, l^{\infty}\right)} \\
& \quad \leq \int_{0}^{t} \Upsilon_{2}(\tau)\left[\left\|z^{(m)}-z^{(m-1)}\right\|_{\left(\tau, l^{\infty}\right)}+\left\|u^{(m+1)}-u^{(m)}\right\|_{\left(\tau, M_{\infty \times n}\right)}\right] d \tau .
\end{aligned}
$$

Write

$$
K^{(m)}(t)=\left\|z^{(m+1)}-z^{(m)}\right\|_{\left(t, l^{\infty}\right)}+\left\|u_{[k]}^{(m+1)}-u_{[k]}^{(m)}\right\|_{\left(t, \mathbb{R}^{n}\right)}, \quad t \in[0, c] .
$$

We deduce from (35), (36) that there is $\Upsilon \in \mathbb{L}\left([0, c], \mathbb{R}_{+}\right)$such that

$$
K^{(m)}(t) \leq \int_{0}^{t} \Upsilon(\tau) K^{(m-1)}(\tau) d \tau, \quad m \geq 1
$$

Set

$$
\left[\left|K^{(m)}\right|\right]=\max \left\{K^{(m)}(t) \exp \left[-2 \int_{0}^{t} \Upsilon(\tau) d \tau\right]: t \in[0, c]\right\} .
$$

Then we have

$$
\begin{aligned}
K^{(m)}(t) & \leq\left[\left|K^{(m-1)}\right|\right] \int_{0}^{t} \Upsilon(\tau) \exp \left[2 \int_{0}^{\tau} \Upsilon(\xi) d \xi\right] d \tau \\
& \leq\left[\left|K^{(m-1)}\right|\right] \exp \left[2 \int_{0}^{t} \Upsilon(\tau) d \tau\right]
\end{aligned}
$$

and consequently

$$
\left[\left|K^{(m)}\right|\right] \leq \frac{1}{2}\left[\left|K^{(m-1)}\right|\right] \text { for } m \geq 1 .
$$

Then $\lim _{m \rightarrow \infty}\left[\left|K^{(m)}\right|\right]=0$ and consequently there are the limits

$$
\tilde{z}(t, x)=\lim _{m \rightarrow \infty} z^{(m)}(t, x), \quad \tilde{u}(t, x)=\lim _{m \rightarrow \infty} u^{(m)}(t, x) \quad \text { uniformly on } E_{c},
$$

where $\tilde{z}=\left\{\tilde{z}_{k}\right\}_{k \in \mathbb{N}}, \tilde{u}=\left[\tilde{u}_{i j}\right]_{i \in \mathbb{N}, 1 \leq j \leq n}$ and $\tilde{u}_{[k]}=\left(\tilde{u}_{k 1}, \ldots, \tilde{u}_{k n}\right)$ for $k \in \mathbb{N}$.

It follows from $\left(C_{m}\right)$ that there exist the derivatives $\partial_{x} \tilde{z}, k \in \mathbb{N}$ and $\partial_{x} \tilde{z}_{k}=\tilde{u}_{[k]}$ for $k \in \mathbb{N}$. 
IV. Now we prove that $\tilde{z}: E_{c} \rightarrow \mathbb{S}$ is a solution to (1), (2). Write $\tilde{g}_{[k]}(\cdot, t, x)=$ $g_{[k]}\left[\tilde{z}, \partial_{x} \tilde{z}\right](\cdot, t, x), \quad(t, x) \in E \cap\left(\left[a_{k}, c\right] \times \mathbb{R}^{n}\right), k \in \mathbb{N}$. It follows from (III) that $\tilde{z}_{k}(t, x)=\psi_{k}(t, x)$ on $E_{0 . k}$ for $k \in \mathbb{N}$ and

$$
\begin{aligned}
\partial_{t} \tilde{z}_{k}(t, x)= & \psi_{k}\left(a_{k}, \tilde{g}_{[k]}\left(a_{k}, t, x\right)\right)+\int_{a_{k}}^{t} F_{k}\left(P\left[\tilde{z}, \partial_{x} \tilde{z}_{k}\right](\tau, t, x)\right) d \tau \\
& \left.-\int_{a_{k}}^{t} \partial_{q} F_{k}\left(P\left[\tilde{z}, \partial_{x} \tilde{z}_{k}\right](\tau, t, x)\right)\right) \circ \partial_{x} \tilde{z}_{k}\left(\tau, \tilde{g}_{[k]}(\tau, t, x)\right) d \tau,
\end{aligned}
$$

where $(t, x) \in E \cap\left(\left[a_{k}, c\right] \times \mathbb{R}^{n}\right), k \in \mathbb{N}$. Suppose that $k \in \mathbb{N}$ is fixed. For given $(t, x) \in$ $E \cap\left(\left[a_{k}, c\right] \times \mathbb{R}^{n}\right)$, let us put $y=\tilde{g}_{[k]}\left(a_{k}, t, x\right)$. It follows that the relations $x=\tilde{g}_{[k]}\left(t, a_{k}, y\right)$ and $y=\tilde{g}_{[k]}\left(a_{k}, t, x\right)$ are equivalent. We conclude from (37) that

$$
\begin{aligned}
& \tilde{z}_{k}\left(t, \tilde{g}_{[k]}\left(t, a_{k}, x\right)\right)=\psi_{k}\left(a_{k}, y\right) \\
& \quad+\int_{a_{k}}^{t} F_{k}\left(\tilde{T}_{k}(\tau, y)\right) d \tau-\int_{a_{k}}^{t} \partial_{q} F_{k}\left(\tilde{T}_{k}(\tau, y)\right) \circ \partial_{x} \tilde{z}_{k}\left(\tau, \tilde{g}_{[k]}\left(\tau, a_{k}, y\right)\right) d \tau
\end{aligned}
$$

where

$$
\tilde{T}_{k}(\tau, y)=\left(\tau, \tilde{g}_{[k]}\left(\tau, a_{k}, y\right), \tilde{z}_{\left(\tau, \tilde{g}_{[k]}\left(\tau, a_{k}, y\right)\right)}, \tilde{z}_{\varphi\left(\tau, \tilde{g}_{[k]}\left(\tau, a_{k}, y\right)\right)}, \partial_{x} \tilde{z}_{k}\left(\tau, \tilde{g}_{[k]}\left(\tau, a_{k}, y\right)\right)\right) .
$$

By differentiating (38) with respect to $t$ and by putting again $x=\tilde{g}_{[k]}\left(t, a_{k}, y\right)$, we obtain that $\tilde{z}$ is a weak solution of (1), (2).

V. It follows form $\left(A_{m}\right)-\left(C_{m}\right)$ that the sequences $\left\{z^{(m)}\right\}$ and $\left\{\partial_{x} z^{(m)}\right\}$ satisfy the conditions

$$
\left\|z^{(m)}\right\|_{\left(t, l^{\infty}\right)} \leq d, \quad\left\|\partial_{x} z^{(m)}\right\|_{\left(t, M_{\infty \times n}\right)} \leq r
$$

and

$$
\left\|\partial_{x} z^{(m)}(t, x)-\partial_{x} z^{(m)}(t, \bar{x})\right\|_{\left(t, M_{\infty \times n}\right)} \leq r_{0}\|x-\bar{x}\|
$$

where $m \in \mathbb{N},(t, x),(t, \bar{x}) \in E_{c}$. From the above inequalities we obtain in the limit, letting $m$ tend to $\infty$, estimates (18), (19).

VI. Now we prove (21). It follows from Assumption $H[F]$ that

$$
\begin{aligned}
& \|\hat{z}-\tilde{z}\|_{\left(t, l^{\infty}\right)}+\left\|\partial_{x} \hat{z}^{(m)}-\partial_{x} \tilde{z}^{(m)}\right\|_{\left(t, M_{\infty \times n}\right)} \\
& \quad \leq\|\psi-\tilde{\psi}\|_{\mathbb{X}}+\int_{0}^{t} \Gamma_{\star}(\tau)\left[\|\hat{z}-\tilde{z}\|_{\left(\tau, l^{\infty}\right)}+\left\|\partial_{x} \hat{z}^{(m)}-\partial_{x} \tilde{z}^{(m)}\right\|_{\left(\tau, M_{\infty \times n}\right)}\right] d \tau, \quad t \in[0, c] .
\end{aligned}
$$

Then we obtain (21) from the Gronwall inequality. This completes the proof of the theorem.

\section{The main result}

Suppose that Assumptions $H[\varphi], H[F]$ are satisfied and $\psi \in \mathbb{X}$. Let us denote by $\Xi[\psi]$ the solution to (1), (2). It follows from Theorem 3.2 that $\Xi[\psi]$ exists on $E_{c}$ and it is unique. Then we have: $\Xi: \mathbb{X} \rightarrow C\left(E_{c}, l^{\infty}\right)$. We will denote by $\mathbb{Y}$ the class of all $\psi \in \mathbb{X}$ satisfying the conditions: 
$\sup \left\{\left\|\psi_{k}\right\|_{E_{0 . k}}: k \in \mathbb{N}\right\}<c_{0}, \quad \sup \left\{\left\|\partial_{x} \psi_{k}\right\|_{E_{0 . k}}: k \in \mathbb{N}\right\}<c_{1}$,

$\sup \left\{\frac{1}{\|x-\bar{x}\|}\left\|\partial_{x} \psi_{k}(t, x)-\partial_{x} \psi_{k}(t, \bar{x})\right\|:(t, x),(t, \bar{x}) \in E_{0 . k}, x \neq \bar{x}, k \in \mathbb{N}\right\}<c_{1}$.

We prove that for each $\psi \in \mathbb{Y}$ there exists the Fréchet derivative $\partial \Xi[\psi]$ of $\Xi$ at the point $\psi$. Moreover, if $\psi \in \mathbb{Y}, \pi \in \mathbb{X}$ and $\overline{\mathbf{z}}=\partial \Xi[\psi] \pi$ then $\overline{\mathbf{z}}$ is a solution of a linear system of integral functional equations generated by (1), (2).

Suppose that Assumptions $H[\varphi], H[F]$ are satisfied. Write

$$
\begin{aligned}
& \hat{\zeta}(t)=c_{0} \exp \left[2 \int_{0}^{t} \beta(\tau) d \tau\right], \\
& \hat{\vartheta}(t)=\hat{d}\left\{\exp \left[\hat{d} \int_{0}^{t} \beta(\tau) d \tau\right]+\int_{0}^{t} \gamma(\mu) \exp \left[\hat{d} \int_{\mu}^{t} \beta(\tau) d \tau\right] d \mu\right\},
\end{aligned}
$$

where $t \in[0, c]$ and

$$
\hat{C}=\hat{\zeta}(c), \quad \hat{d}=\exp \left[\bar{C} \int_{0}^{c} \gamma(\tau) d \tau\right] \max \left\{c_{1}, 2 \bar{c} \hat{C}, 1+Q_{0}\right\}
$$

Suppose that $\pi \in \mathbb{X}, \pi=\left\{\pi_{k}\right\}_{k \in \mathbb{N}}$. Let us denote by $C_{\pi . c}[\hat{\zeta}, \hat{\vartheta}]$ the class of all $z: E_{c} \rightarrow l^{\infty}$, $z=\left\{z_{k}\right\}_{k \in \mathbb{N}}$, satisfying the conditions:

(i) $z \in C\left(E_{c}, l^{\infty}\right)$ and $z_{k}(t, x)=\pi_{k}(t, x)$ for $(t, x) \in E_{0 . k}$ and $k \in \mathbb{N}$,

(ii) $\|z\|_{\left(t, l^{\infty}\right)} \leq \hat{\zeta}(t)$ for $t \in[0, c]$ and

$$
\sup \left\{\frac{1}{\|x-\bar{x}\|}\|z(\tau, x)-z(\tau, \bar{x})\|_{\infty}:(\tau, x),(\tau, \bar{x}) \in E_{c}, \tau \leq t\right\} \leq \hat{\vartheta}(t) \text { for } t \in[0, c] .
$$

In this section we denote by $z(\cdot ; \psi)=\left\{z_{k}(\cdot ; \psi)\right\}_{k \in \mathbb{N}}$ the solution to (1), (2). Let us consider the Cauchy problem

$\omega^{\prime}(\tau)=-\partial_{q} F_{k}\left(\tau, \omega(\tau),(z(\cdot ; \psi))_{(\tau, \omega(\tau))},(z(\cdot ; \psi))_{\varphi(\tau, \omega(\tau))}, \partial_{x} z(\tau, \omega(\tau) ; \psi)\right), \quad \omega(t)=x$,

where $(t, x) \in E \cap\left(\left[a_{k}, c\right] \times \mathbb{R}^{n}\right)$ and $k \in \mathbb{N}$. The solution to (40) will be denote by $g_{[k]}[\psi](\cdot, t, x)$. If Assumptions $H[\varphi], H[F]$ are satisfied and $\psi \in \mathbb{X}$ then for each $k \in \mathbb{N}$ the solution $g_{[k]}[\psi](\cdot, t, x)$ is defined on $\left[a_{k}, t\right]$. For $k \in \mathbb{N}$ we put

$$
\begin{aligned}
& T_{k}[\psi](\tau, t, x) \\
& =\left(\tau, g_{[k]}[\psi](\tau, t, x),(z(\cdot ; \psi))_{\left(\tau, g_{[k]}[\psi](\tau, t, x)\right)},(z(\cdot ; \psi))_{\varphi\left(\tau, g_{[k]}[\psi](\tau, t, x)\right)},\right. \\
& \left.\quad \times \partial_{x} z\left(\tau, g_{[k]}[\psi](\tau, t, x) ; \psi\right)\right) .
\end{aligned}
$$

Suppose that $\pi \in \mathbb{X}, \pi=\left\{\pi_{k}\right\}_{k \in \mathbb{N}}$, and $z \in C_{\pi . c}[\hat{\zeta}, \hat{\vartheta}], z=\left\{z_{k}\right\}_{k \in \mathbb{N}}$. Let us denote by $W[z]=\left\{W_{k}[z]\right\}_{k \in \mathbb{N}}$ the function defined by

$$
W_{k}[z](t, x)=\pi_{k}(t, x) \quad \text { for }(t, x) \in E_{0 . k}
$$


and

$$
\begin{aligned}
& W_{k}[z](t, x)=\pi_{k}\left(a_{k}, g_{[k]}[\psi]\left(a_{k}, t, x\right)\right)+\int_{a_{k}}^{t} \partial_{v} F_{k}\left(T_{k}[\psi](\tau, t, x)\right) \diamond z_{\left(\tau, g_{[k]}[\psi](\tau, t, x)\right)} d \tau \\
& +\int_{a_{k}}^{t} \partial_{w} F_{k}\left(T_{k}[\psi](\tau, t, x)\right) \diamond z_{\varphi\left(\tau, g_{[k]}[\psi](\tau, t, x)\right)} d \tau \text { for }(t, x) \in E \cap\left(\left[a_{k}, c\right] \times \mathbb{R}^{n}\right), \quad
\end{aligned}
$$

where $k \in \mathbb{N}$. We consider the linear system of integral functional equations

$$
z=W[z]
$$

Lemma 4.1 Suppose that Assumptions $H[\varphi], H[F]$ are satisfied and $\pi \in \mathbb{X}$. Then there exists exactly one solution $\overline{\mathbf{z}}: E_{c} \rightarrow l^{\infty}$ of (43) and

$$
\begin{aligned}
& \|\overline{\mathbf{z}}\|_{\infty} \leq \hat{C} \quad \text { on } E_{c}, \\
& \|\overline{\mathbf{z}}(t, x)-\overline{\mathbf{z}}(t, \bar{x})\|_{\infty} \leq \hat{L}\|x-\bar{x}\| \quad \text { on } E_{c}
\end{aligned}
$$

where $\hat{C}$ is given by (39) and $\hat{L}=\hat{\vartheta}(c)$.

Proof We prove that

$$
W: C_{\pi . c}[\hat{\zeta}, \hat{\vartheta}] \rightarrow C_{\pi . c}[\hat{\zeta}, \hat{\vartheta}]
$$

It follows from Assumptions $H[\varphi], H[F]$ that for $z \in C_{\pi . c}[\hat{\zeta}, \hat{\vartheta}],(t, x) \in E_{c}, \tau \leq t$, we have

$$
\left|W_{k}[z](\tau, x)\right| \leq c_{0}+2 \int_{0}^{t} \beta(\mu) \hat{\zeta}(\mu) d \mu=\hat{\zeta}(t)
$$

and consequently

$$
\|W[z]\|_{\left(t, l^{\infty}\right)} \leq \hat{\zeta}(t) \text { for } t \in[0, c] .
$$

For the above $z$ and for $(\tau, x),(\tau, \bar{x}) \in E_{c}, \tau \leq t$, we have

$$
\begin{aligned}
\left|W_{k}[z](\tau, x)-W_{k}[z](\tau, \bar{x})\right| & \leq \hat{d}\left[1+\int_{0}^{t} \gamma(\mu) d \mu+\int_{0}^{t} \beta(\mu) \hat{\vartheta}(\mu) d \mu\right]\|x-\bar{x}\| \\
& =\hat{\vartheta}(t)\|x-\bar{x}\|, \quad k \in \mathbb{N} .
\end{aligned}
$$

This gives

$$
\|W[z](\tau, x)-W[z](\tau, \bar{x})\|_{\infty} \leq \hat{\vartheta}(t)\|x-\bar{x}\|,
$$

where $(\tau, x),(\tau, \bar{x}) \in E_{c}, \tau \leq t$. From (47), (48) we deduce (46).

For $z, z_{\star} \in C_{\pi . c}[\hat{\zeta}, \hat{\vartheta}]$ and for $k \in \mathbb{N}$ we have

$$
W_{k}[z](t, x)-W_{k}\left[z_{\star}\right](t, x)=0 \quad \text { on } E_{0 . k},
$$

and

$$
\left|W_{k}[z](t, x)-W_{k}\left[z_{\star}\right](t, x)\right| \leq 2 \int_{0}^{t} \beta(\tau)\left\|z-z_{\star}\right\|_{\left(\tau, l^{\infty}\right)} d \tau \quad \text { on } E \cap\left(\left[a_{k}, c\right] \times \mathbb{R}^{n}\right) .
$$


Write

$$
\left[\left|z-z_{\star}\right|\right]=\max \left\{\left\|z-z_{\star}\right\|_{\left(\tau, l^{\infty}\right)} \exp \left[-4 \int_{0}^{t} \beta(\tau) d \tau\right]: t \in[0, c]\right\} .
$$

It follows from (49), (50) that for $z, z_{\star} \in C_{\pi . c}[\hat{\zeta}, \hat{\vartheta}]$ we have

$$
\left[\left|W[z]-W\left[z_{\star}\right]\right|\right] \leq \frac{1}{2}\left[\left|z-z_{\star}\right|\right] .
$$

From the Banach fixed point theorem we deduce that there is exactly one solution $\overline{\mathbf{z}} \in$ $C_{\pi . c}[\hat{\zeta}, \hat{\vartheta}]$ of (43). We conclude from (46) that conditions (44), (45) are satisfied. This completes the proof.

Theorem 4.2 Suppose that Assumptions $H[\varphi], H[F]$ are satisfied. Then

1. for each $\psi \in \mathbb{Y}$ there exists the Fréchet derivative $\partial \Xi[\psi]$,

2. if $\psi \in \mathbb{Y}, \pi \in \mathbb{X}$ and $\overline{\mathbf{z}}=\partial \Xi[\psi] \pi, \overline{\mathbf{z}}=\left\{\overline{\mathbf{z}}_{k}\right\}_{k \in \mathbb{N}}$, then $\overline{\mathbf{z}}$ is a solution to (43) with $W$ given by (41), (42).

Proof The proof will be divided into three steps.

I. Let $\psi \in \mathbb{Y}$ and $\pi \in \mathbb{X}$ be fixed. There is $\varepsilon_{0}>0$ such that for $\xi \in I_{0}=\left(-\varepsilon_{0}, \varepsilon_{0}\right)$ we have $\psi+\xi \pi \in \mathbb{X}$. Let $\Delta_{\xi}: E_{c} \rightarrow l^{\infty}, \xi \in I_{0}, \xi \neq 0$, be defined by

$$
\Delta_{\xi}=\left\{\Delta_{\xi . k}\right\}_{k \in \mathbb{N}}, \quad \Delta_{\xi . k}(t, x)=\frac{1}{\xi}\left[z_{k}(t, x ; \psi+\xi \pi)-z_{k}(t, x ; \psi)\right], \quad k \in \mathbb{N} .
$$

It follows from Lemma 4.1 that there is exactly one solution $\overline{\mathbf{z}}=\left\{\overline{\mathbf{z}}_{k}\right\}_{k \in \mathbb{N}}$ to (43), $\overline{\mathbf{z}} \in$ $C\left(E_{c}, l^{\infty}\right)$ and $\overline{\mathbf{z}}$ satisfies (44), (45). We prove that

$$
\lim _{\xi \rightarrow 0}\left\|\Delta_{\xi}(t, x)-\overline{\mathbf{z}}(t, x)\right\|_{\infty}=0 \quad \text { uniformly on } E_{c} .
$$

II. It follows from Theorem 3.2 that for $k \in \mathbb{N}, \xi \in I_{0}, \xi \neq 0$ we have

$$
\begin{aligned}
\partial_{t} \Delta_{\xi . k}(t, x) \\
=\frac{1}{\xi}\left\{F_{k}\left(t, x,(z(\cdot ; \psi+\xi \pi))_{(t, x)},(z(\cdot ; \psi+\xi \pi))_{\varphi(t, x)}, \partial_{x} z_{k}(t, x ; \psi+\xi \pi)\right)\right. \\
\quad-F_{k}\left(t, x,(z(\cdot ; \psi))_{(t, x)},(z(\cdot ; \psi))_{\varphi(t, x)}, \partial_{x} z_{k}(t, x ; \psi)\right) \text { on } E \cap\left(\left[a_{k}, c\right] \times \mathbb{R}^{n}\right),
\end{aligned}
$$

and

$$
\Delta_{\xi . k}(t, x)=\pi_{k}(t, x) \text { on } E_{0 . k} .
$$

Set

$$
\bar{Q}_{k}(t, x ; \psi)=\left(t, x,(z(\cdot ; \psi))_{(t, x)},(z(\cdot ; \psi))_{\varphi(t, x)}, \partial_{x} z_{k}(t, x ; \psi)\right), \quad k \in \mathbb{N},
$$

and

$$
\Theta_{k}[\psi, \pi](t, x ; \xi, \eta)=(1-\eta) \bar{Q}_{k}(t, x ; \psi)+\eta \bar{Q}_{k}(t, x ; \psi+\xi \pi), \quad k \in \mathbb{N},
$$


where $\eta \in[0,1]$. Then we obtain from (52) that

$$
\begin{aligned}
& \partial_{t} \Delta_{\xi . k}(t, x)=\int_{0}^{1} \partial_{v} F_{k}\left(\Theta_{k}[\psi, \pi](t, x ; \xi, \eta)\right) \diamond\left(\Delta_{\xi}\right)_{(t, x)} d \eta \\
& \quad+\int_{0}^{1} \partial_{w} F_{k}\left(\Theta_{k}[\psi, \pi](t, x ; \xi, \eta)\right) \diamond\left(\Delta_{\xi}\right)_{\varphi(t, x)} d \eta \\
& \quad+\int_{0}^{1} \partial_{q} F_{k}\left(\Theta_{k}[\psi, \pi](t, x ; \xi, \eta)\right) \circ \partial_{x} \Delta_{\xi . k}(t, x) d \eta, \quad(t, x) \in E \cap\left(\left[a_{k}, c\right] \times \mathbb{R}^{n}\right),
\end{aligned}
$$

where $k \in \mathbb{N}$. Let us denote by $g_{[k]}[\psi, \pi ; \xi](\cdot, t, x)$ the solution of the Cauchy problem

$$
\omega^{\prime}(\tau)=-\int_{0}^{1} \partial_{q} F_{k}\left(\Theta_{k}[\psi, \pi](\tau, \omega(\tau) ; \xi, \eta)\right) d \eta, \quad \omega(t)=x,
$$

where $(t, x) \in E \cap\left(\left[a_{k}, c\right] \times \mathbb{R}^{n}\right)$. Write

$$
Q_{k}[\psi, \pi ; \xi, \eta](\tau, t, x)=\Theta_{k}[\psi, \pi]\left(\tau, g_{[k]}[\psi, \pi ; \xi](\tau, t, x) ; \xi, \eta\right), \quad k \in \mathbb{N} .
$$

It follows from (54) that $\Delta_{\xi}$ satisfies relations

$$
\begin{aligned}
& \Delta_{\xi . k}(t, x)=\pi_{k}\left(a_{k}, g_{[k]}[\psi, \pi ; \xi]\left(a_{k}, t, x\right)\right) \\
& +\int_{a_{k}}^{t} \int_{0}^{1} \partial_{v} F_{k}\left(Q_{k}[\psi, \pi ; \xi, \eta](\tau, t, x)\right) \diamond\left(\Delta_{\xi}\right)_{\left(\tau, g_{[k]}[\psi, \pi ; \xi](\tau, t, x)\right)} d \eta d \tau \\
& +\int_{a_{k}}^{t} \int_{0}^{1} \partial_{w} F_{k}\left(Q_{k}[\psi, \pi ; \xi, \eta](\tau, t, x)\right) \diamond\left(\Delta_{\xi}\right)_{\varphi\left(\tau, g_{[k]}[\psi, \pi ; \xi](\tau, t, x)\right)} d \eta d \tau
\end{aligned}
$$

where $(t, x) \in E \cap\left(\left[a_{k}, c\right] \times \mathbb{R}^{n}\right)$ and $k \in \mathbb{N}$.

III. We construct an integral functional inequality for $\Delta_{\xi}-\overline{\mathbf{z}}$. Write

$$
\begin{aligned}
& A_{k}(t, x)=\pi_{k}\left(a_{k}, g_{[k]}[\psi, \pi ; \xi]\left(a_{k}, t, x\right)\right)-\pi_{k}\left(a_{k}, g_{[k]}[\psi]\left(a_{k}, t, x\right)\right), \\
& B_{k}(t, x)=\int_{0}^{t} \int_{0}^{1} \partial_{v} F_{k}\left(Q_{k}[\psi, \pi ; \xi, \eta](\tau, t, x)\right) \diamond\left(\Delta_{\xi}-\overline{\mathbf{z}}\right)_{\left(\tau, g_{[k]}[\psi, \pi ; \xi](\tau, t, x)\right)} d \eta d \tau \\
& +\int_{0}^{t} \int_{0}^{1} \partial_{w} F_{k}\left(Q_{k}[\psi, \pi ; \xi, \eta](\tau, t, x)\right) \diamond\left(\Delta_{\xi}-\overline{\mathbf{z}}\right)_{\varphi\left(\tau, g_{[k]}[\psi, \pi ; \xi](\tau, t, x)\right)} d \eta d \tau
\end{aligned}
$$


and

$$
\begin{aligned}
& C_{k}(t, x)=\int_{a_{k}}^{t} \int_{0}^{1} \partial_{v} F_{k}\left(Q_{k}[\psi, \pi ; \xi, \eta](\tau, t, x)\right) \diamond\left[\overline{\mathbf{z}}_{\left(\tau, g_{[k]}[\psi, \pi ; \xi](\tau, t, x)\right)}\right. \\
& \times-\overline{\mathbf{z}}_{\left.\left(\tau, g_{[k]}[\psi](\tau, t, x)\right)\right] d \eta d \tau} \\
& +\int_{a_{k}}^{t} \int_{0}^{1} \partial_{w} F_{k}\left(Q_{k}[\psi, \pi ; \xi, \eta](\tau, t, x)\right) \diamond\left[\overline{\mathbf{z}}_{\varphi\left(\tau, g_{[k]}[\psi, \pi ; \xi](\tau, t, x)\right)}-\overline{\mathbf{z}}_{\left.\varphi\left(\tau, g_{[k]}[\psi](\tau, t, x)\right)\right] d \eta d \tau}\right. \\
& D_{k}(t, x)=\int_{a_{k}}^{t} \int_{0}^{1}\left[\partial_{v} F_{k}\left(Q_{k}[\psi, \pi ; \xi, \eta](\tau, t, x)\right)-\partial_{v} F_{k}\left(T_{k}[\psi](\tau, t, x)\right)\right] \\
& \quad \times \diamond \overline{\mathbf{z}}_{\left(\tau, g_{[k]}[\psi](\tau, t, x)\right)} d \eta d \tau \\
& \quad+\int_{a_{k}}^{t} \int_{0}^{1}\left[\partial_{w} F_{k}\left(Q_{k}[\psi, \pi ; \xi, \eta](\tau, t, x)\right)-\partial_{w} F_{k}\left(T_{k}[\psi](\tau, t, x)\right)\right] \diamond \overline{\mathbf{z}}_{\varphi\left(\tau, g_{[k]}[\psi](\tau, t, x)\right)} d \eta d \tau
\end{aligned}
$$

where $k \in \mathbb{N}$. Then we have

$$
\Delta_{\xi . k}(t, x)-\overline{\mathbf{z}}_{k}(t, x)=A_{k}(t, x)+B_{k}(t, x)+C_{k}(t, x)+D_{k}(t, x), \quad k \in \mathbb{N} .
$$

It follows from Assumption $H[F]$ and from (21) that

$$
\begin{aligned}
& \left\|g_{[k]}[\psi, \pi ; \xi](\tau, t, x)-g_{[k]}[\psi](\tau, t, x)\right\| \\
& \quad \leq \bar{C}\left|\int_{\tau}^{t} \gamma(\mu)\left\|g_{[k]}[\psi, \pi ; \xi](\mu, t, x)-g_{[k]}[\psi](\mu, t, x)\right\| d \mu\right|+|\xi| A_{\star}, \quad k \in \mathbb{N},
\end{aligned}
$$

where

$$
A_{\star}=3\|\pi\|_{\mathbb{X}} \exp \left[\int_{0}^{c} \Gamma_{\star}(\tau) d \tau\right] \int_{0}^{c} \gamma(\tau) d \tau .
$$

This gives

$$
\left\|g_{[k]}[\psi, \pi ; \xi](\tau, t, x)-g_{[k]}[\psi](\tau, t, x)\right\| \leq|\xi| \hat{A}, \quad k \in \mathbb{N}
$$

where

$$
\hat{A}=A_{\star} \exp \left[\bar{C} \int_{0}^{c} \gamma(\tau) d \tau\right]
$$

and consequently

$$
\left|A_{k}(t, x)\right| \leq c_{1} \hat{A}|\xi|, \quad k \in \mathbb{N} .
$$

It is clear that

$$
\left|B_{k}(t, x)\right| \leq 2 \int_{0}^{t} \beta(\tau)\left\|\Delta_{\xi}-\overline{\mathbf{z}}\right\|_{\left(\tau, l^{\infty}\right)} d \tau, \quad k \in \mathbb{N} .
$$


We conclude from Assumption $H[F]$ and from (45) that

$$
\begin{aligned}
\left|C_{k}(t, x)\right| & \leq \hat{L}\left(1+Q_{0}\right) \int_{a_{k}}^{t} \beta(\tau)\left\|g_{[k]}[\psi, \pi ; \xi](\tau, t, x)-g_{[k]}[\psi](\tau, t, x)\right\| d \tau \\
& \leq|\xi| \hat{L}\left(1+Q_{0}\right) \int_{0}^{c} \beta(\tau) d \tau \hat{A}, \quad k \in \mathbb{N} .
\end{aligned}
$$

It follows from Assumption $H[F]$ and from (21) that the expressions

$$
\begin{aligned}
& \int_{a_{k}}^{t} \int_{0}^{1}\left\|\partial_{v} F_{k}\left(Q_{k}[\psi, \pi ; \xi, \eta](\tau, t, x)\right)-\partial_{v} F_{k}\left(T_{k}[\psi](\tau, t, x)\right)\right\|_{\infty ; \star} d \eta d \tau, \\
& \int_{a_{k}}^{t} \int_{0}^{1}\left\|\partial_{w} F_{k}\left(Q_{k}[\psi, \pi ; \xi, \eta](\tau, t, x)\right)-\partial_{w} F_{k}\left(T_{k}[\psi](\tau, t, x)\right)\right\|_{\infty ; \star} d \eta d \tau, \quad k \in \mathbb{N},
\end{aligned}
$$

can be estimated from above by

$$
\bar{C} \int_{a_{k}}^{t} \gamma(\tau)\left\|g_{[k]}[\psi, \pi ; \xi](\tau, t, x)-g_{[k]}[\psi](\tau, t, x)\right\| d \tau+|\xi| \hat{B}, \quad k \in \mathbb{N},
$$

where

$$
\hat{B}=3 \int_{0}^{c} \gamma(\tau) d \tau\|\pi\|_{\mathbb{X}} \exp \left[\int_{0}^{c} \Gamma_{\star}(\tau) d \tau\right] .
$$

We conclude from the above relations and from (44), (57) that

$$
\left|D_{k}(t, x)\right| \leq|\xi| \hat{D}, \quad k \in \mathbb{N},
$$

where

$$
\hat{D}=2 \hat{C}\left[\bar{C} \hat{A} \int_{0}^{c} \gamma(\tau) d \tau+\hat{B}\right]
$$

It follows from (56) and (58)-(61) that there is $\hat{Q} \in \mathbb{R}_{+}$such that

$$
\left\|\Delta_{\xi}-\overline{\mathbf{z}}\right\|_{\left(t, l^{\infty}\right)} \leq|\xi| \hat{Q}+2 \int_{0}^{c} \beta(\tau)\left\|\Delta_{\xi}-\overline{\mathbf{z}}\right\|_{\left(\tau, l^{\infty}\right)} d \tau, \quad t \in[0, c] .
$$

By the Gronwall inequality we obtain

$$
\left\|\Delta_{\xi}-\overline{\mathbf{z}}\right\|_{\left(t, l^{\infty}\right)} \leq|\xi| \hat{Q} \exp \left[2 \int_{0}^{c} \beta(\tau) d \tau\right], \quad t \in[0, c] .
$$

This completes the proof of (51).

The assertion of the theorem follows from (51).

Remark 4.3 It is easy to see that Theorems 3.2 and 4.2 can be applied to problems (4), (2) and (8), (2). 
Open Access This article is distributed under the terms of the Creative Commons Attribution License which permits any use, distribution, and reproduction in any medium, provided the original author(s) and the source are credited.

\section{References}

1. Augustynowicz, A., Kamont, Z.: On Kamke's functions in uniqueness theorems for first order partial differential functional equations. Nonlinear Anal. TMA 14, 837-850 (1990)

2. Bassanini, P., Turo, J.: Generalized solutions to free boundary problems for hyperbolic systems of functional partial differential equations. Ann. Mat. Pura Appl. 156, 211-230 (1990)

3. Besala, P.: Observations on quasi-linear partial differential equations. Ann. Polon. Math. 53, 267-283 (1991)

4. Brandi, P., Ceppitelli, R.: Existence, uniqueness and continuous dependence for a first order nonlinear partial differential equations in a hereditary structure. Ann. Polon. Math. 47, 121-135 (1986)

5. Brandi, P., Marcelli, C.: Haar inequality in hereditary setting and applications. Rend. Sem. Mat. Univ. Padova 96, 177-194 (1996)

6. Byszewski, L.: Finite systems of strong nonlinear differential functional degenerate implicit inequalities with first order partial derivatives. Univ. Iagell. Acta Math. 29, 77-84 (1992)

7. Cinquini Cibrario, M.: Sopra una class di sistemi de equazioni nonlineari a derivate parziali in piú variabili indipendenti. Ann. Mat. pura ed appl. 140, 223-253 (1985)

8. Czernous, W.: Infinite systems of first order PFDEs with mixed conditions. Ann. Polon. Math. 94, 209-230 (2008)

9. Człapiński, T.: On the mixed problem for quasilinear partial differential - functional equations of the first order. Z. Anal. Anwend. 16, 463-478 (1997)

10. Hale, J.K., Verdun, L., Sjoerd, M.: Introduction to Functional Differential Equations. Springer, Berlin (1993)

11. Kamont, Z.: Infinite systems of hyperbolic functional differential equations. Ukrainian Math. J. 55(12), 2006-2030 (2003)

12. Kamont, Z.: Hyperbolic Functional Differential Inequalities. Kluwer Academic Publishers, Dordrecht (1999)

13. Kamont, Z.: Infinite systems of hyperbolic functional differential inequalities. Nonlinear Anal. TMA 51, $1429-1445$ (2002)

14. Kamont, Z.: Existence of solutions to Hamilton-Jacobi functional differential equations. Nonl. Anal. TMA 73, 767-778 (2010)

15. Kępczyńska, A.: Implicit difference methods for quasilinear differential functional equations on the Haar pyramid. Z. Anal. Anvend. 27, 213-231 (2008)

16. Przạdka, K.: Difference methods for non-linear partial differential functional equations of the first order. Math. Nachr. 138, 105-123 (1988)

17. Puźniakowska-Gałuch, E.: Implicit difference methods for nonlinear first order partial functional differential systems. Appl. Math (Warsaw) 37, 459-482 (2010)

18. Szarski, J.: Cauchy problem for an infinite system of differential functional equations with first order partial derivatives (Special issue dedicated to Władysław Orlicz on the occasion of his seventy-fifth birthday). Comm. Math., special issue I, 293-300 (1978)

19. Szarski, J.: Comparison theorems for infinite systems of differential functional equations and strongly coupled infinite systems of first order partial differential equations. Rocky Mt. J. Math. 10, 237-246 (1980)

20. Topolski, K.A.: On the existence of viscosity solutions for the functional-differential cauchy problem. Ann. Soc. Math. Polon. Comment. Math. 39, 207-223 (1999)

21. Topolski, K.A.: On the vanishing viscosity method for first order differential-functional IBVP. Czechosl. Math. J. 58, 927-947 (2008)

22. Turo, J.: Nonlocal problems for first order functional partial differential equations. Ann. Polon. Math. 72, 99-114 (1999)

23. Ważewski, T.: Sur le probléme de Cauchy relatif á un systéme d'équations aux dériveés partielles. Ann. Soc. Polon. Math. 15, 101-127 (1936)

24. Wu, J.: Theory and Applications of Partial Functional Differential Equations. Springer, Berlin (1996)

25. Zubik-Kowal, B.: The method of lines for first order partial differential functional equations. Studia Sci. Math. Hungar. 34, 413-428 (1998) 